\title{
Search Costs in Consumer Product Choice: Does Delaying the Provision of Information increase Choice Efficiency?
}

\author{
By Axel Sonntag
}

School of Economics and CBESS, University of East Anglia

\begin{abstract}
Using online price comparison and shopping platforms makes experiencing slow connections, lags and waiting times for information an unfortunate reality. However, little attention has been paid to analyzing the effects of delayed provision of information on product choice behavior. In this article, I introduce an information processing model to derive search pattern predictions and test them in a multi-attribute choice laboratory experiment. Information requested during a search process was not provided immediately but after short time delays. Increasing these waiting times reduced the amount of looked-up information but did not affect choice quality. It seems that high time delays nudged decision-makers to think twice about how much and what kind of information should be looked-up, whereas low time delays induced inefficient over-searching. As technological advances are expected to further reduce Internet delay times, the observed change in search behavior could affect the profitability of manufacturers' production and retailers' advertising strategies.
\end{abstract}

\section{JEL classification codes C91, D11, D12, D81, D83 \\ Keywords}

Search costs, time delays, multi-attribute consumer product choice, outcome quality, choice efficiency

Centre for Behavioural and Experimental Social Science University of East Anglia Norwich Research Park Norwich NR4 7TJ United Kingdom www.uea.ac.uk/ssf/cbess 


\title{
Search Costs in Consumer Product Choice: Does Delaying the Provision of Information increase Choice Efficiency?
}

\author{
Axel Sonntag*
}

March 2013

\begin{abstract}
Using online price comparison and shopping platforms makes experiencing slow connections, lags and waiting times for information an unfortunate reality. However, little attention has been paid to analyzing the effects of delayed provision of information on product choice behavior. In this article, I introduce an information processing model to derive search pattern predictions and test them in a multi-attribute choice laboratory experiment. Information requested during a search process was not provided immediately but after short time delays. Increasing these waiting times reduced the amount of looked-up information but did not affect choice quality. It seems that high time delays nudged decision-makers to think twice about how much and what kind of information should be looked-up, whereas low time delays induced inefficient over-searching. As technological advances are expected to further reduce Internet delay times, the observed change in search behavior could affect the profitability of manufacturers' production and retailers' advertising strategies.
\end{abstract}

Keywords: search costs, time delays, multi-attribute consumer product choice, outcome quality, choice efficiency

JEL-codes: C91, D11, D12, D81, D83

*University of East Anglia, School of Economics and Centre for Behavioural and Experimental Social Sciences, email: a.sonntag@uea.ac.uk 


\section{Introduction}

In the pre-Internet era, searching for information to make an informed product choice came at substantial cost. Nowadays it is not information scarcity, but quite the contrary, the excessive amount of available information that makes choice costly in the first place. Considering that processing huge amounts of information can take a lot of time, already very short time delays per piece of information could accumulate to search costs of formidable size. However, the effects of delaying the provision of information on product choice behavior are largely unknown. That comes as a surprise as waiting times for information (lags) are a natural characteristic of online environments which have become an integral part of our daily lives. ${ }^{1}$ As such time delays might change information search behavior and the resulting product choices considerably, it is of vital importance to both manufacturers and online retailers to know how much and what kind of information their potential customers actually use to choose a product, when they experience time delays in online shopping platforms. This paper is the first to analyze the effect of different levels of time delay on (i) how consumers search for information, (ii) how accurate they make product choices and (iii) how efficient they use information in a multi-attribute choice environment. ${ }^{2}$ After recording process data on information acquisition behavior in an incentivized Mouselab setting (first stage), a choice-based conjoint analysis (second stage) enabled me to calculate subjective attribute weights and utility values for the products used in stage one. Thus, in addition to other studies that addressed behavioral search pattern changes, I was able to analyze the important dimensions of outcome quality and choice efficiency as well.

My results indicate that decision-makers seemed to adapt to higher time delays by reducing the number of revealed attributes, focusing on less than half of the available information elements. Complementarily, lower waiting times induced search on product characteristics that were disregarded under high time delays. Although different levels of time delay did not affect choice quality at all, the significant change in information processing behavior could potentially determine which combination of product characteristics would be chosen more likely. Therefore, the profitability of

\footnotetext{
${ }^{1}$ This is especially true for the growing sector of online shopping via mobile devices, where response times usually are still of tedious magnitude.

${ }^{2}$ In this paper, choice quality is a relative measure of utility derived from the actually chosen product divided by the utility derived from the best available product. Choice efficiency is defined as the level of choice quality divided by the number of looked-up elements. See section 2 for details.
} 
the managerial decision, whether to concentrate manufacturing and sales resources on products that outperform their direct competitors on only a few attributes, or instead produce models that are more balanced across their attribute values, also depends on the speed of information provision of the online retailer. As technological advances are expected to continue reducing delay times of online retailing platforms in the future, more balanced products could be more successful as compared to those that are only superior regarding a few characteristics.

\section{Determinants of information search behavior}

The notion that human decision-makers adapt their processing strategies to the underlying choice environment has a long tradition in decision research (Simon 1956, Payne 1976). Such adaptive decision-makers (Payne et al. 1993) were found to change their information search and processing behavior by a variety of different choice-environmental characteristics, such as task complexity (Payne 1976, Caplin et al. 2011) or payoff structures (Payne et al. 1988, Bröder 2003). For the purpose of this study, however, the effects of search costs, especially in the form of exogenous search time manipulations, are of primary interest.

\section{Time pressure and the opportunity costs of time}

Although research has paid little attention to the effects of non-monetary search costs in the form of time delays so far, many studies have investigated the effects of time pressure on information processing behavior. As a result of increased time pressure, Payne et al. (1988) found that participants used a more attribute-wise ${ }^{3}$ information search which indicates the use of non-compensatory ${ }^{4}$ strategies. Smith et al. (1982), who found similar patterns, explained this behavior by the decisionmakers' reduced confidence of being able to apply complex strategies correctly under

\footnotetext{
${ }^{3}$ Search is deemed 'attribute-wise' or 'within-attributes' if information is looked-up in a sequence such that one attribute of all alternatives is revealed (e.g. $C P U$-speed of all notebook computers in the choice set) before the next attribute of all alternatives is processed (e.g. RAM-capacity of the notebooks), i.e. searching 'one attribute after the other'. Conversely, search is deemed 'alternative-wise' or 'within-alternatives' if the information of all attributes of one alternative is revealed (e.g. all characteristics of notebook 1) before information on all attributes of the next alternative is searched (e.g. notebook 2), i.e. 'searching one alternative after the other'.

${ }^{4} \mathrm{~A}$ decision strategy is deemed 'compensatory' if the bad (good) values of an alternative on one attribute can be compensated by good (bad) values on other attributes. Conversely, if a strategy does not allow such an inter-attribute off-set, it is deemed 'non-compensatory'.
} 
time pressure. In addition to searching in a more attribute-wise manner, decisionmakers also seemed to have searched for less information (Payne et al. 1988), and did so more selectively (Verplanken and Weenig 1993, Rieskamp and Hoffrage 2008). Interestingly, when being confronted with an explicit time limit and the participants 'see the clock ticking', some authors found an increase in processing speed instead of less information search, i.e. subjects did not reduce the number of information elements but seemed to process them faster (Verplanken 1993, Verplanken and Weenig 1993) resulting in less processing time per element (Dhar and Nowlis 1999). In contrast to imposing a constraining time limit directly, Payne et al. (1996) incentivized the opportunity costs of time monetarily by specifying the experimental payoff as a monotonically falling function in decision time, i.e. participants got paid less if they deliberated longer. Qualitatively, their results do not differ from studies using direct time pressure. Similarly, Rieskamp and Hoffrage (2008), comparing choice- versus session-wise time constraints (with one session containing many choices), found only very small differences between their time cost paradigms. Hence, increasing the decision-makers' freedom to allocate time between single choices more flexibly did not affect their tendency to use more non-compensatory strategies in environments with high opportunity costs of time. Although the above studies manipulated the dimension of time to make search costly in an indirect manner, none of them implemented time-wise search costs directly, which would most closely resemble search costs in real online environments provided by Internet retailers.

\section{Time delays}

A direct way of implementing time-wise search costs experimentally is by imposing time delays on the provision of information. However, this has rarely been done so far. Urbany (1986) implemented such a time delay paradigm and found an inverse relationship of search costs and the amount of acquired information; however, he used a combination of time delays and monetary search costs and could therefore not distinguish the pure effect of time delays from the combined effect. Nevertheless, he suggested that future research should put "more emphasis on time and frustration costs" (Urbany 1986, p.270). Although Urbany cannot be expected to have anticipated how important the world wide web would become, his research suggestion is more relevant than ever. However, to my knowledge, only one other study has investigated the effect of delay times so far. In a controlled field experimental 
setting, Schurman and Brutlag (2009) analyzed the effect of server delay times on the frequency of search queries at the Internet search portals of Google and bing. They found that reducing the speed of delivering search results could have severe consequences on the competitiveness of search service providers. Stepwise increasing the server response times from $50 \mathrm{~ms}$ to $2000 \mathrm{~ms}$ statistically significantly decreased the number of search queries, the user satisfaction, the time until the next click, and finally the revenue per user. Considering the heavy use of online price comparison and shopping environments that make diverse time delays and waiting times an unfortunate (daily) reality, stresses the need for research on the effect of time delays on information processing, especially in multi-attribute choice situations. Hence, by using pure time delays, this paper attempts to bridge that research gap. Furthermore, besides analyzing behavioral search pattern changes, this paper investigates how well consumers adapt their choice behavior (i.e. how time delays affect the quality of choice outcomes) as well.

\section{Choice quality}

Only a few experimental studies that either varied monetary search costs or time pressure also analyzed the quality ${ }^{5}$ of the resulting choice outcome. In this paper, I will define choice quality, similar to Zakay and Wooler (1984), Johnson and Payne (1985), Coupey and Narayanan (1996), and Chu and Spires (2000), to be a relative measure of utility induced by the actually chosen alternative divided by the potential utility derived from the best available product of a certain choice set (see section 2 for details). Note that such an approach requires a specific experimental set-up which will be discussed in detail in section 3 .

Payne et al. (1988) found that time pressure had a clear negative impact on choice quality and similarly Zakay and Wooler (1984, p279) report a "destructive effect on decision effectiveness". Although Urbany (1986) did not mention choice quality explicitly, his results indicate that the joint search costs of monetary charges and delay times had a quality decreasing effect for situations of high uncertainty and narrow price dispersion. ${ }^{6}$ High search costs caused less information searching,

\footnotetext{
${ }^{5}$ What I will call choice quality throughout this paper has also been referred to as "choice accuracy" (e.g. Johnson and Payne 1985) or "decision effectiveness" (Zakay and Wooler 1984) in the decision-making literature.

${ }^{6}$ In their setting, where consumers had to shop for consumer products (homogeneous within a specific product category and brand), not choosing the cheapest alternative available can be considered as non-optimal choice quality.
} 
which in an environment with low price dispersion led to lower choice quality. ${ }^{7}$ When investigating different forms of information provision, Benbasat and Dexter (1986) revealed that under high time pressure a graphic problem representation helped decision-makers to achieve outcomes of considerable quality. However, when there was no time pressure, information presented in tables (and tables in combination with graphics) resulted in higher quality than graphics alone. This suggests that decision-makers are not per se unwilling to process detailed information, as long as they can explore the choice environment at their own pace.

Recent evidence by Caplin et al. (2011) indicates that the complexity of choice problems negatively affects choice quality. As decision aid systems were found to not only reduce decision effort but also potentially improve choice quality (Chu and Spires 2000), such tools could possibly be applied more effectively in complex environments. However, Diehl (2005) reports that tools meant to aid decision-makers could also have side effects that are detrimental to choice quality. In principle, environments where all alternatives were ordered by expected utility (best at the top) should help to identify the most suitable option more quickly. Nevertheless, under low search costs, decision-makers search 'too many' products further down the list, finally resulting in a lower choice quality. Hence, whether increasing search costs would lower or even improve choice quality very much depends on the underlying choice environment. Along the same lines, Gigerenzer and Brighton (2009) also found a significant 'less is more' effect, i.e. using heuristic choice rules that deliberately use less information could substantially improve choice quality. However, it is still unknown how search costs in the form of delaying the provision of information affect choice quality. Therefore, this study investigates the effects of different levels of time delay on information search behavior and choice quality.

In section 2 of this paper I will briefly review related strategy selection models and introduce the Effort-Certainty model from which I subsequently derive testable hypotheses. Section 3 describes the experimental design, before section 4 presents the empirical results. Section 5 discusses the results and section 6 concludes the main findings of this paper.

\footnotetext{
${ }^{7}$ This relationship will be discussed in more detail in section 2 as a feature of lexicographic search.
} 


\section{Modeling Adaptive Choice}

In the currently prevailing framework of adaptive choice, decision-makers are assumed to use an Effort-Accuracy (E-A) trade-off to choose a decision rule from a portfolio of available strategies (Johnson and Payne 1985, Payne et al. 1988, 1993). Essentially, strategies that require more decision effort are expected to result in higher choice accuracy. However, Klein and Yadav (1989) and later Kuo et al. (2004) tested the E-A trade-off empirically and found no and only very limited support for such a relationship, respectively. Moreover, recently, research on fast and frugal heuristics provided both theoretical and empirical evidence that the E-A trade-off assumption does not always hold (Czerlinski et al. 1999, Gigerenzer and Brighton 2009, Katsikopoulos et al. 2010, Kruglanski and Gigerenzer 2011). Sometimes, using the right strategy in the right environment even increases accuracy by simultaneously reducing decision effort. Hence, I propose a different cost-benefit model that is particularly tailored to analyzing purchasing decisions regarding durable consumer products.

\section{The Effort-Certainty Model}

Consider a consumer who wants to buy a specific durable consumer product, e.g. a notebook computer. In such situation, even before starting to search for a concrete product, the consumer usually has a general knowledge about the domain of notebook computers, i.e. she has a rough idea what she can do with notebooks, how much they cost on average, how long she could use a model until it needs to be replaced by a new one etc. Even in the case that it would be the first time of purchasing a notebook computer, she might have at least some understanding about its importance to her. Let us call the perceived importance of a product domain $I(d)$ with $d$ being the product domain. Thus, how important a product choice situation is perceived depends on the product domain $d$.

Let us assume that the consumer fears being disappointed by choosing a product that does not fulfill her expectations, as well as regretting to have forgone a better alternative (Loomes and Sugden 1982, 1986). ${ }^{8}$ As gaining more information about

\footnotetext{
${ }^{8}$ Since Loomes and Sugden presented their theory of regret (1982) and disappointment (1986) aversion, both concepts have found extensive repercussions in the literature. For an application to non-lottery decisions in the consumer context see Tsiros and Mittal (2000), Inman and Zeelenberg (2002), or Abendroth and Diehl (2006).
} 
the available choice set reduces uncertainty, and thereby the chance of selecting an alternative that could result in disappointment or regret, the decision-maker should consequently search for as much information as possible. However, as there are opportunity costs (e.g. of time), even presumably costless search comes at a cost by which the amount of information acquisition will be limited. If $l$ represents the number of looked-up pieces of information, $E(l)$ denotes the total amount of search effort. Also, let $C(l)$ be a measure for perceived certainty, i.e. the perceived degree of knowledge about the decision environment. ${ }^{9}$ In that case, the decision-maker is expected to adapt her information search to a decision situation such that

$$
I(d) \cdot C(l)-E(l) \geq 0
$$

Thus, a decision-maker is assumed to adapt the amount of looked-up information such that the degree of knowledge about a decision environment $C(l)$ weighted by its perceived importance $I(d)$ always remains greater than or equal to the overall decision effort $E(l)$. Note that the left-hand-side of (1) is not meant to be maximized as it would be assumed in standard economic models of search (e.g. Stigler 1961). Instead, I only assume that a decision-maker chooses to search any number of information elements such that the associated search effort does not exceed the utility from better knowing the decision environment. ${ }^{10}$ Overall, the decision-maker is expected to process more information for choices in product domains that are perceived to be more important.

Keeping this general notion of the Effort-Certainty model in mind, adding a few more details to the model will derive concrete hypotheses later on. In (1), a decisionmaker was assumed to have a level of perceived importance that varies by product domain $d$. More specifically, it is not the product domain as such but average anticipated values of specific characteristics of a product domain that cause specific levels of perceived importance. Let $j$ be one of a total $J$ dimensions to fully describe a product domain (e.g. $j=$ average price level) and $X_{d j}$ be a concrete realization of the characteristic value of dimension $j$ for product domain $d$ (e.g. $X_{d j}=\$ 1200$ ). If the decision-maker is in a position to identify which dimension is more or less important to her, she could weight the set of characteristic values $\boldsymbol{X}_{d j}=\left\{X_{d 1}, X_{d 2}, \ldots, X_{d J}\right\}$

\footnotetext{
${ }^{9} C(l)$ could also be understood as the confidence of knowing the decision environment.

${ }^{10}$ Requiring that the net-utility of information acquisition always has to be positive could be interpreted similar to Simon's aspiration level thinking (Simon 1956).
} 
with a corresponding set of weights $\boldsymbol{\beta}_{j}=\left\{\beta_{1}, \beta_{2}, \ldots, \beta_{J}\right\} .{ }^{11}$ Then the perceived importance of a product domain can be defined as $I(d)=\sum_{j} \beta_{j} X_{d j}$. In that sense, expensive and cheap notebook computers are considered to be two different product domains specified by different characteristic values $\boldsymbol{X}_{d j}$ (e.g. price levels) that are weighted by the same set of weights $\boldsymbol{\beta}_{j}$. Following this notion, the situation of purchasing expensive notebook computers is expected to be considered more important than purchasing rather cheap notebook computers.

The attribute values and weights considered above $\left(X_{d j}, \beta_{j}\right)$ describe differences across product domains. In contrast, the next paragraph will introduce a different set of attribute values and weights that are used to compare products within a product domain (e.g. one cheap notebook computer vs. another cheap notebook computer).

Figure 1: Illustration of the Effort-Certainty model for varying product domains and search costs
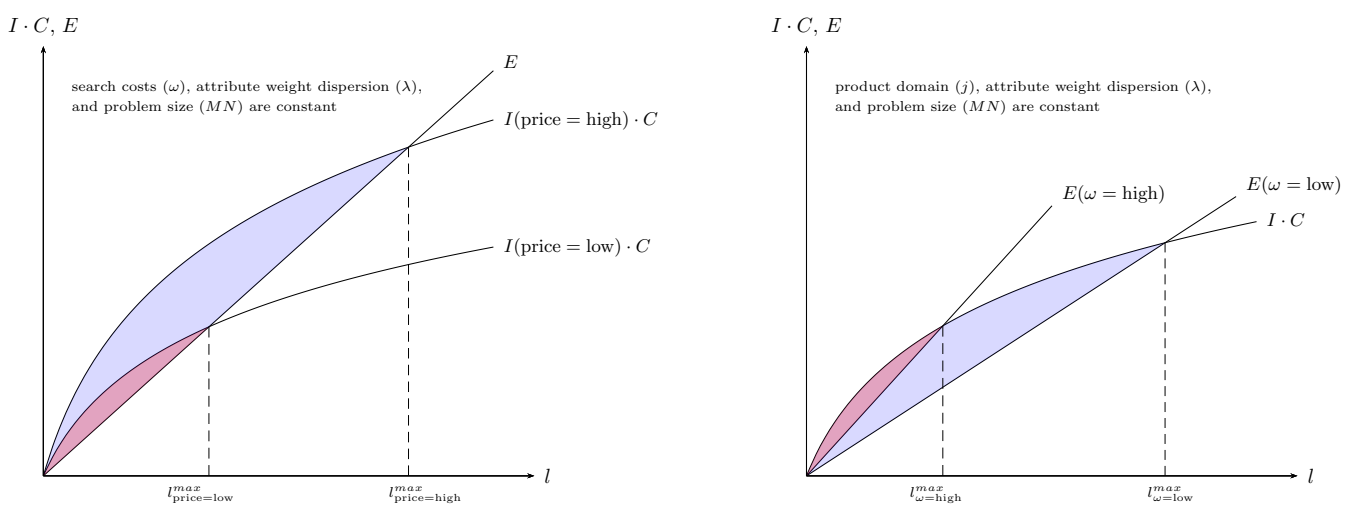

Notes: Figure 1 graphically illustrates the principle idea of the Effort-Certainty framework for the case of varying product importance (left) and search costs (right) with the $l^{\max }$ locations on the horizontal axis marking the upper bound for the demand for information (see equation (4)), dependent on the importance of the product domain and search costs, respectively.

Let a product domain consist of $N$ different products that are described by $M$ attributes each. The maximum amount of information that can be acquired is then defined by $M \cdot N$, the problem size, and $l /(M N)$ describes the relative share of looked-up information elements. Consider further that not all pieces of information might be equally important to the decision-maker. Some product characteristics

\footnotetext{
${ }^{11}$ Note that the weights $\boldsymbol{\beta}_{j}$ are only individual- and not domain-specific. Thus, a decision-maker is expected to apply a constant set of weights to all possible product choice situations.
} 
could be more important than others (e.g. the price could be considered more important than the color of a notebook computer). Let $Y_{m}$ describe a specific value of attribute $m$ (e.g. ' $\$ 1000$ ' for the attribute 'price') and $\gamma_{m}$ be the relative weight a decision-maker attaches to $m .{ }^{12}$ Then choosing product $n$ described by the product specific vector of characteristic values $\boldsymbol{Y}_{n}=\left\{Y_{n 1}, Y_{n 2}, \ldots, Y_{n M}\right\}$ provides the decision-maker with utility $u_{n}$.

$$
u_{n}=\sum_{m=1}^{M} \gamma_{m} Y_{n m}
$$

I assume that decision-makers look-up information elements of the most important attribute first. Consequently, for any given distribution of attribute weights $\gamma_{m}=\left\{\gamma_{1}, \gamma_{2}, \ldots, \gamma_{M}\right\}$ that is ordered, such that $\gamma_{m} \geq \gamma_{m+1} \forall m \in[1, M-1]$ decision-makers are expected to reveal all elements of the attribute $m$ before starting to process information on the attribute $m+1$. In line with (2), it is reasonable to look-up elements that contain attribute values of the most important attribute first (i.e. $\gamma_{1}=\max _{m \in M} \gamma_{m}$ ) as their marginal impact on $u_{n}$ is potentially greater than the marginal impact of revealing information on the second most important attribute, which itself is expected to have a higher marginal impact than the third most important attribute and so on. This rationale is also known as lexicographic search order (see Tversky 1972).

The more information decision-makers have about a specific choice problem, the more confident they become to choose the 'right' product. ${ }^{13}$ Therefore, looking-up information on more important attributes (e.g. price) is expected to increase the perceived certainty $C$ by a greater extent than looking-up less important bits of information (e.g. color). Thus, the average marginal gains from looking-up additional information are expected to fall and the perceived certainty for the general case is defined as $C(l, M, N, \lambda)=\left(\frac{l}{M N}\right)^{1-\lambda}$ with $\lambda \in[0,1]$ being a measure of the dispersion of attribute weights. ${ }^{14}$ If all product characteristics were of equal importance, the dispersion of attribute weights, and therefore $\lambda$, would be zero and $C$ simplified to

\footnotetext{
${ }^{12} \gamma_{m} \in[0,1]$ and $\sum_{m=1}^{M} \gamma_{m}=1$

${ }^{13}$ Right in this context means best fitting to the decision-makers' preferences, i.e. $u=$ $\max _{n \in N} u_{n}$.

${ }^{14}$ More precisely, $\lambda=\sum_{i=1}^{M-1}\left(\gamma_{i}-\gamma_{i+1}\right)$. Although the marginal returns from information search are expected to fall in a stepwise manner (on the transition from more important to less important attribute values), I use a simplified functional form that is not only concave but also differentiable in $l$ such that $\frac{\partial C}{\partial l}>0$ and $\frac{\partial^{2} C}{\partial l^{2}}<0$.
} 
$l /(M N)$, the relative share of looked-up information elements. Further considering that looking-up one piece of information requires an effort of $\omega$, the overall search effort can be written as $E(l, \omega)=l \cdot \omega$. Rewriting (1) for the general case then becomes

$$
\sum_{j} \beta_{j} X_{d j} \cdot\left(\frac{l}{M N}\right)^{1-\lambda}-l \omega \geq 0
$$

In order to make predictions about how different decision environments affect the amount of information search, equation (3) is solved for $l$ and subsequently differentiated with respect to the search costs per piece of information and the attribute value of one arbitrary characteristic of the product domain, $\omega$ and $X_{d j}$, respectively. ${ }^{15}$

$$
l \leq \frac{\left(\sum_{j} \beta_{j} X_{d j}\right)^{\frac{1}{\lambda}} \cdot(M N)^{\frac{\lambda-1}{\lambda}}}{\omega^{\frac{1}{\lambda}}}
$$

As $M, N, \sum_{i} \beta_{j} X_{d j}, \omega$, and $\lambda$ per definition contain positive values, $\partial l / \partial \omega<0$. Consequently, an increase in decision effort results in lower demand for reducing uncertainty. This result is also depicted on the right-hand-side of Figure 1 where the maximum amount of looked-up information $l^{\max }$ is reduced (shifted to the left) as search costs increase. On the contrary, increasing the characteristic value of a dimension that is directly proportional to the perceived importance of a product domain (i.e. $\beta_{j}>0$ ), ceteris paribus, is expected to result in more search, thus $\partial l / \partial X_{d j}>0 .{ }^{16}$ The resulting consequences are displayed on the left-hand-side of Figure 1. Assuming that the average price level of a product domain positively affects its perceived importance (i.e. $\beta_{\text {price }}>0$ ), an increase in the average price level would cause more search (shifting $l^{\max }$ to the right).

\footnotetext{
${ }^{15} X_{d j} \in \boldsymbol{X}_{d j}$. Note that $X_{d j}$ contains the characteristic value of one dimension (e.g. average price level) that, together with others, define a product domain $d$ (e.g. notebook computers). Differentiating $l$ with respect to $X_{d j}$ thus analyzes how changing the characteristic value of dimension $j$ affects the looked-up amount of information.

${ }^{16}$ Alternatively, $\beta_{j}<0$ would result in $\partial l / \partial X_{d j}<0$.
} 


\section{Hypotheses}

Based on the Effort-Certainty model and considering the way of presenting the choice set experimentally (see Figure 2), the following hypotheses with respect to search patterns and intensity as well as choice quality are derived. Remember that the perceived importance of a specific choice environment depends on the characteristics of the product domain $X_{d j}$. As shown above $\partial l / \partial X_{d j}>0 \forall \beta_{j}>0$, any changes in characteristic values that would increase the level of perceived importance $I$ are expected to increase the amount of looked-up information. Therefore, by choosing average product price and average perceived necessity as two product domain characteristics $j$, hypotheses $\mathrm{H} 1$ and $\mathrm{H} 2$ are stated. Assuming that the importance of a product domain increases with the anticipated price level, i.e. $\beta_{\text {price }}>0$, of its products, this results in:

Hypothesis 1: The higher the average price of a product domain is expected to be, the more information will be acquired.

Likewise, assuming that the importance of a product domain increases with the anticipated level of everyday necessity, i.e. $\beta_{\text {necessity }}>0$, this leads to:

Hypothesis 2: The higher the perceived dependence on (a product of) a specific product domain is, the more information will be acquired.

Similarly to the changes in perceived importance $I$, so also changes in search costs $\omega$ are expected to affect information search patterns and intensity. However, as $\partial l / \partial \omega<0$, an increase in search costs is expected to reduce the amount of information acquired.

Hypothesis 3: The more costly the information search is perceived, the less information will be acquired.

It is a well-established empirical result that both monetary search costs and time pressure decrease the amount of acquired information (see section 1). Consistently, the Effort-Certainty model predicts a reduction in information search for increasing search costs. One standard measure to compare search patterns in the decision making literature is Payne's search index (1976). By distinguishing withinattribute (wATT) from within-alternative (wALT) transitions in information search, the search index is defined as $S I=($ wALT - wATT $) /($ wALT + wATT $)$ and thus $S I \in[-1,1] .{ }^{17}$ By the specific way of looking-up information every decision can be

\footnotetext{
${ }^{17}$ For a definition of alternative-wise and attribute-wise transitions, see also footnote 3 .
} 
described by one of four search patterns: attribute-wise $(S I<0)$, alternative-wise $(S I>0)$, in a balanced $(S I=0)$ or in a random pattern. ${ }^{18}$ Given a constant number of attributes and alternatives, reducing the number of searched information elements $\left(l^{\prime \prime}<l^{\prime}\right)$ would, conditional on the applied search pattern, result in either lower, equal or higher levels of the search index measure. Therefore, it is possible to use observable differences in the search index measure (if any) to infer the search pattern applied by decision-makers.

$$
\text { If } l^{\prime \prime}<l^{\prime} \Rightarrow \begin{cases}S I^{\prime \prime}<S I^{\prime} & \text { attribute-wise search pattern } \\ S I^{\prime \prime}=S I^{\prime} & \text { balanced or random search pattern } \\ S I^{\prime \prime}>S I^{\prime} & \text { alternative-wise search pattern }\end{cases}
$$

As I assume a lexicographic search order (i.e. information elements are searched attribute-wise, starting with the most important attribute) I expect that high search costs, on average, will result in lower observed values of SI. Due to the chosen form of information presentation in an 8 (attributes) $\times 12$ (alternatives) matrix, a lexicographic search order implies that SI increases in the number of elements searched. ${ }^{19}$ Consequently, as increased search costs are expected to decrease the amount of information search, SI is expected to decrease in search costs as well.

Hypothesis 4: The more costly information search is perceived, the lower the search index measure will be (more attribute-wise search patterns).

Regarding the utility that a decision-maker gains from choosing a specific product (as defined in (2)), I define decision quality $\theta$ as a relative measure of utility derived from the actually chosen product divided by the utility derived from the best possible product choice. Choice quality thus is defined as $\theta=u_{\text {actual }} / u_{\max }$. Given a structured ${ }^{20}$ choice environment, the decision quality $\theta$ is a concave function of the

\footnotetext{
${ }^{18}$ Whether random search results in a positive or negative search index depends on the number of attributes and alternatives. Actually, $S I<0 \forall M<N$ and $S I>0 \forall M>N$. Note that the search index is an unbiased measure of the search patterns for this experiment, as the ratio of the number of attributes $M$ and alternatives $N$ is constant throughout all treatment conditions. For a discussion of this property see Böckenholt and Hynan (1994).

${ }^{19}$ If a lexicographic search order is used, reducing the amount of searched information only means reducing the number of looked-up attributes (but continuing to look-up this reduced set of attributes from all alternatives). Thus, revealing less information necessarily results in a shift from alternative-wise to attribute-wise transitions which due to the very definition of the search index results in lower values of SI.

${ }^{20}$ I refer to a decision environment as structured if the attributes vary in their importance to the
} 
amount of information searched before the choice is made. Hence, every additional bit of information increases choice quality, with decreasing marginal gains. ${ }^{21}$ Therefore, any decrease in the amount of looked-up information is expected to decrease choice quality. Considering simultaneously that the Effort-Certainty model predicts a detrimental effect of search costs on the amount of revealed information leads to the prediction that increased search costs are expected to decrease choice quality.

Hypothesis 5: The more costly information search is perceived, the lower the resulting choice quality will be.

However, whether or not a reduction in decision quality is substantial depends on the weight of the information elements that are not revealed due to increased search costs. Which elements are disregarded first depends on the applied search order. Remember that a lexicographic search order would start by ignoring the least important bits of information first, thereby resulting only in a marginal decrease in choice quality. Therefore, finding evidence in line with H5 could be triggered by two conditions: either the dispersion in attribute weights was rather small ${ }^{22}$ or decisionmakers did not use a lexicographic search order. Conversely, the same two conditions would have to be fulfilled simultaneously in order to reject H5. The decision-makers had to use a lexicographic search order and the dispersion in attribute weights had to be sufficiently large. Therefore, rejecting H5 (i.e. an increase in search costs does not decrease choice quality) provides evidence for the use of a lexicographic search order.

However, usually decision-makers do not only care about a reasonable choice quality, but also about how much effort they need to put forward in order to achieve a certain level of quality. It is therefore interesting to investigate choice efficiency as well. In this paper I define choice efficiency $\eta$ as the level of choice quality divided by the number of looked-up elements, thus $\eta=\theta / l$. When applying a lexicographic search order, information elements that are revealed first should have a greater influence on choice quality than those revealed later on. Therefore, reducing the amount of information search should result in a reduction of choice quality, but choice quality is expected to be reduced to a relatively lesser extent than the amount of information

decision-maker and thus if the variance of attribute weights is greater than zero and consequently $\lambda>0$.

$21 \frac{\partial \theta}{\partial l}>0$ and $\frac{\partial^{2} \theta}{\partial l^{2}}<0, \forall l \leq M N$

${ }^{22}$ If the variance in attribute weights is rather small, even using a lexicographic search order and thereby disregarding only the least important attribute(s) has a strong impact on decision quality. 
itself. $^{23}$ Consequently, the measure of choice efficiency as defined above should decrease in the amount of information searched. Considering simultaneously that the Effort-Certainty model predicts a detrimental effect of search costs on the amount of information revealed, leads to the prediction that increased search costs are expected to increase choice efficiency.

Hypothesis 6: The more costly information search is perceived, the higher the resulting choice efficiency will be.

However, whether or not the increase in choice efficiency is substantial depends on the weight of the dismissed information elements and therefore on the applied search order and the dispersion in attribute weights. Two conditions must be fulfilled in order to observe results in line with H6: the decision-makers have to use a lexicographic search order and the dispersion in attribute weights has to be sufficiently large. If only one condition is not met, H6 needs to be rejected. Therefore, observing results in line with $\mathrm{H} 6$ can also be interpreted as support for the assumption that a lexicographic search order is used.

\section{Experimental Design}

\section{Information Presentation and Process Tracing}

This study follows the commonly used Mouselab approach of displaying potentially useful information in attributes $\times$ alternatives matrices (see Johnson et al. 1986, Payne et al. 1993, Lee and Lee 2004, Birnbaum and Schmidt 2008, Willemsen and Johnson 2011). Especially, the unbroken boom of online shopping where matrix displays are regularly used for product comparisons (Riedl and Brandstätter 2007, Shao et al. 2008) as well as the natural way of searching by using a computer mouse to reveal information supports a Mouselab setting (Reisen et al. 2008). Thus, for the purpose of this experiment I only included products that are commonly known to be available in online shops. The experiment was implemented with Mouselab WEB (Willemsen and Johnson 2004, 2011). ${ }^{24}$

Moving the mouse cursor over a matrix element removed its cover (temporarily)

\footnotetext{
${ }^{23}$ This is true for a non-zero variance in product attribute weights.

${ }^{24}$ This open-source PHP/HTML/MySQL package is especially convenient for implementing time delays.
} 
Figure 2: Screenshot of the Mouselab experiment: selection of 'Expensive Notebook Computers'

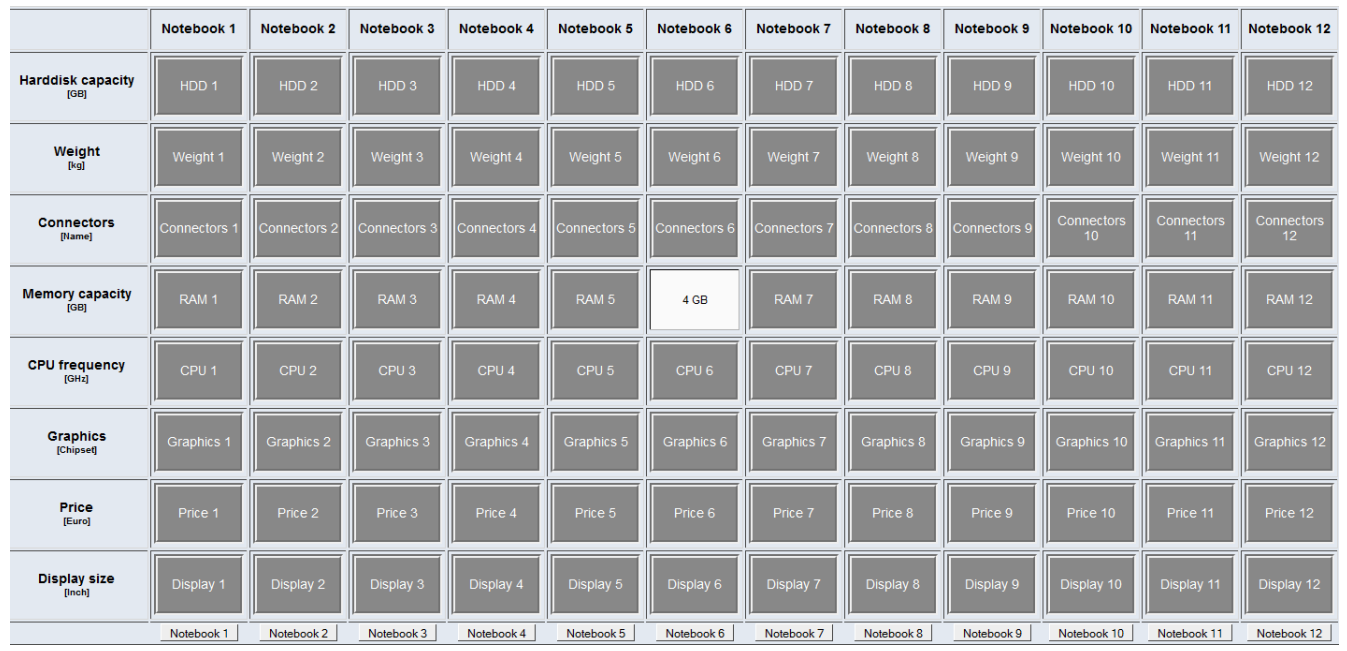

Twelve products (columns) are defined by eight attributes (rows) each. All cells are covered unless the mouse cursor is moved over them to reveal the underlying attribute value. Only one cell can be opened at a time.

and its underlying value could be considered for decision making (e.g. the element Memory capacity of Notebook 6, see Figure 2). However, once the mouse cursor left an element (e.g. to move to and uncover another element), the underlying information of the former element was immediately covered again. Consequently, only one element per time could be viewed. This procedure thus indicated which element was processed at a specific period of time and allowed for tracing the information acquisition process.

\section{Treatment design}

The experimental conditions were varied along three dimensions: search costs (4 levels) were altered between subjects, whereas product necessity (2 levels) and product price (2 levels) were varied within subject. ${ }^{25}$ Hence, all participants were randomly allocated to one of four levels of search costs. Within each search cost treatment they were asked to choose one product from each of the four choice sets presented to them successively. The order of the four choice sets was counterbalanced over the full sample of participants such that every product domain appeared once to be the

\footnotetext{
${ }^{25}$ The results of a pilot classroom experiment (undergraduate students, $n=183$ ) suggested that product price, perceived necessity, purchasing interval and whether a product is a durable or not were relevant dimensions of how important a product choice situation was perceived.
} 
first, second, third or last product domain to be searched in the Mouselab setting.

\section{Search costs}

I used time delays on information acquisition to implement a notion of search costs that, in an era of Internet shopping, regarding external validity seemed to be more appropriate than e.g. monetary search costs. In the no-delay treatment, all cell covers were removed immediately once the mouse cursor entered a matrix element. However, in the delay treatments, participants who moved their mouse cursor over an element had to wait for 330, 660 or 1000 milliseconds (ms) until the underlying information was revealed. Inducing different levels of time delay was expected to change the perceived costs of search considerably (see Hypotheses 3, 4, 5 \& 6).

\section{Product necessity}

Notebook computers and TV sets were chosen as a high and low necessity product category, respectively. As students often own notebooks themselves they should be familiar with that kind of product and know what characteristics they like or dislike when they are asked to choose one. However, selecting TV sets as the second product was not trivial, as they needed to be as similar as possible to notebooks in all dimensions except for the perceived importance in the participants' everyday lives (see Hypothesis 2). ${ }^{26}$

\section{Product price}

It was important to choose two levels of price ranges at which notebooks and TV sets are actually being sold. Therefore, a range of $€ 400-€ 700$ (approx. \$510-\$890) and $€ 1000-€ 1300$ (approx. \$1270-\$1660) were chosen for the low and high product price condition, respectively (see Hypothesis 1$).{ }^{27}$

\footnotetext{
${ }^{26}$ The two product categories should not differ regarding the potential general demand, provide the opportunity to measure attribute levels objectively, be gender-neutral, be reasonably available at the same typical price level, and the matrix presentation form should be perceived natural to a similar degree. However, simultaneously they should potentially differ in perceived necessity. In the line with the notion that notebooks should be perceived more important than TV sets, Zillow.com (2010), in a large consumer survey, found that people before they make their final purchase decision on average spent 4 hours on searching for information on computers whereas they only invested 2 hours in the case of TV sets.

${ }^{27}$ The experimentally used product specifications were not generated artificially, but looked-up at the websites of big retailers of consumer electronics in order to provide real-world, up-to-date product characteristics for the choice problem and to avoid unrealistic combinations of attribute
} 


\section{Incentivizing search behavior}

In order to create an incentive compatible product choice situation, one choice round of one participant was randomly selected after finishing all experimental sessions (see Cox et al. 2011). This participant received a voucher that granted a $50 \%$ subsidy in case of purchasing exactly the product chosen in the 'winning round' (e.g. a specific laptop model) and had four weeks to redeem it. This incentive was supposed to support honest selection behavior along personal preferences. However, as granting a subsidy could potentially induce a bias towards the selection of more expensive products, only $50 \%$ of the actual product price was provided as a potential payoff. ${ }^{28}$

\section{Participants and Procedures}

Each participant had to come to the laboratory twice. In the first session they were provided with the experimental instructions and could familiarize themselves with the Mouselab environment by searching a trial matrix that contained information about a different product domain than those used during the actual experiment. Afterwards, they started the sequence of four $(8 \times 12)$ product choices with additional instructions before each matrix which explained what kind of product domain they were going to choose from the next. Subsequently, they answered an on-screen questionnaire before receiving a participation fee for part one. In the second session the participants engaged in twenty-four binomial product choices. ${ }^{29}$ Each of the forty-eight products was specified by carefully varied levels of the same attributes that defined the products used in the Mouselab part. The resulting choice data was used to perform a choice-based conjoint analysis to elicit attribute weights that subsequently were used to calculate utility values for each product in the Mouselab choice set (see equation (2)). This procedure enabled me to analyze the quality

values which can be a potential issue for artificially constructed commodities.

${ }^{28}$ Although providing a $50 \%$ discount also incentivizes selecting the most expensive model in a narrow utility maximizing sense, this 'strategy' becomes much less salient, as compared to a $100 \%$ discount rate. Actually, participants chose the most expensive products in only $3.2 \%$ of all Mouselab choice settings. The ratio of the price of the actually chosen product to the price of the most expensive product in the choice set did not vary over delay treatments (Kruskal-Wallis test $p=0.963)$.

${ }^{29}$ In order to reduce potential carry-over effects of product knowledge from the Mouselab search to the binomial choice task, at least twenty-four hours cool-off period was required between session one and two. 
of choice outcomes in the Mouselab stage of the experiment. ${ }^{30}$ As all participants were required to participate in both the first and the second part of the experiment, attribute weights, utility values and choice qualities could be calculated at the individual level.

Eighty-four undergraduate students from the Vienna University of Economics and Business (Austria) participated in the experiment. The first and the second part of the experiment on average lasted 45 and 25 minutes, respectively. In addition to participation fees ( $€ 8$ and $€ 4$ for the first and the second part, respectively) participants on average earned $€ 3.7$ from an incentivized ambiguity aversion task at the end of part two, resulting in a total average payment of $€ 15.7$ (approx. \$20). As the search cost treatment was conducted between subjects, the participants were randomly assigned to the $0 \mathrm{~ms}, 330 \mathrm{~ms}, 660 \mathrm{~ms}$ or $1000 \mathrm{~ms}$ delay treatment, resulting in $21,21,23$ and 19 subjects per condition, respectively. ${ }^{31}$

\section{Results}

\section{Testing the hypotheses}

RESULT 1: The product price level did not significantly affect the amount of revealed information.

EvidEnCE: Neither for the number of unique elements, nor for all elements (including repeated look-ups per information element) was there any significant difference between high and low product cost environments. Whereas in the low cost condition an average of 67 (median: 70) unique elements were revealed, in the high cost environment on average 67 (median: 72) were looked-up. This difference was not statistically significant (one-sided Wilcoxon Matched-Pairs Signed-Ranks Test, henceforth osMPWT, $p=0.410) \cdot{ }^{32}$

\footnotetext{
${ }^{30}$ In order to increase the statistical power of the conjoint analysis, but at the same time keep the number of choices per participant manageable without inducing boredom effects, in part two, each participant was assigned to make the decisions for either notebooks or TV sets. Consequently, I could calculate the attribute weights for two (out of four) product domains only, which reduced the number of observations for analyzing choice quality and choice efficiency from (see Tables $2 \& 3$ ).

${ }^{31}$ This design would have resulted in eighty-four decision processes per product domain. However, due to a software error, a total of twenty-one observations had to be removed resulting in sixty-four expensive TV set and eighty-three expensive notebook observations. Testing the distribution across delay treatments with respect to reported gross income and age revealed no significant differences
} 
Table 1: Descriptive statistics

\begin{tabular}{lllllll}
\hline & & $\mathrm{n}$ & elements & search index & choice quality & choice efficiency \\
\hline \multirow{4}{*}{$\begin{array}{llllll}\text { Delay } \\
\text { times }\end{array}$} & 0ms & 84 & $81(89)$ & $-0.339(-0.418)$ & $0.649(0.639)$ & $0.146(0.139)$ \\
& $660 \mathrm{~ms}$ & 91 & $69(72)$ & $-0.375(-0.451)$ & $0.643(0.653)$ & $0.204(0.186)$ \\
& $1000 \mathrm{~ms}$ & 56 & $60(60)$ & $-0.444(-0.490)$ & $0.644(0.652)$ & $0.241(0.194)$ \\
\multirow{4}{*}{$\begin{array}{l}\text { Product } \\
\text { domains }\end{array}$} & cheap TV & 84 & $55(51)$ & $-0.492(-0.491)$ & $0.741(0.766)$ & $0.275(0.265)$ \\
& cheap NB & 84 & $65(67)$ & $-0.449(-0.456)$ & $0.708(0.652)$ & $0.221(0.194)$ \\
& expensive TV & 64 & $65(70)$ & $-0.389(-0.469)$ & $0.719(0.851)$ & $0.243(0.190)$ \\
& expensive NB & 83 & $69(70)$ & $-0.366(-0.474)$ & $0.711(0.766)$ & $0.210(0.200)$ \\
\hline
\end{tabular}

Notes: mean values per treatment, medians in parentheses, n: number of observations, elements: number of uniques elements viewed, search index: measure of information processing pattern as defined on page 12, choice quality: normalized measure of outcome utility, choice efficiency: normalized measure of choice quality divided by the number of unique element viewed, $0 \mathrm{~ms}-1000 \mathrm{~ms}$ : time to wait until requested information is actually revealed

RESULT 2: The product type did not significantly affect the amount of revealed information.

EVIDENCE: In the low necessity treatments (TV sets), on average 64 (median: 64) unique information elements were revealed, whereas participants looked-up on average 69 (median: 69) unique elements when they chose between laptop computers (high necessity). While this difference was statistically significant (osWMPT, $p=0.007$ ), controlling for other variables in the regression analysis resulted in an insignificant coefficient for product type $(p=0.155)$. In addition, although the product type did not affect the number of acquired information elements, participants seemed to use a slightly more alternative-wise search pattern when comparing notebook computers than they used for TV sets (see Table 2).

RESULT 3: Waiting times significantly reduced the amount of processed information.

EVIDENCE: In the baseline treatment (no time delay), participants on average opened 81 (median 84) of 96 totally available elements. Increasing the time delay to 330ms led to significantly less search (one-sided Wilcoxon Signed-Ranks Test, henceforth osWT, $p=0.015) .{ }^{33}$ While participants on average still looked-up 69

(Kruskal-Wallis test $p=0.921$ and $p=0.585$, respectively).

${ }^{32}$ Note that the cost treatment was conducted within subject, i.e. single observations were not independent. Therefore a osMPWT was applied on subject averages per cost treatment. Thus, instead of four observations, one high- and one low-cost average were used per subject.

${ }^{33} \mathrm{As}$ the four observations per participant were not independent, all non-parametric tests were conducted on subject averages unless stated otherwise. 
Figure 3: Information acquisition: number of unique elements and repetition ratios by delay treatments
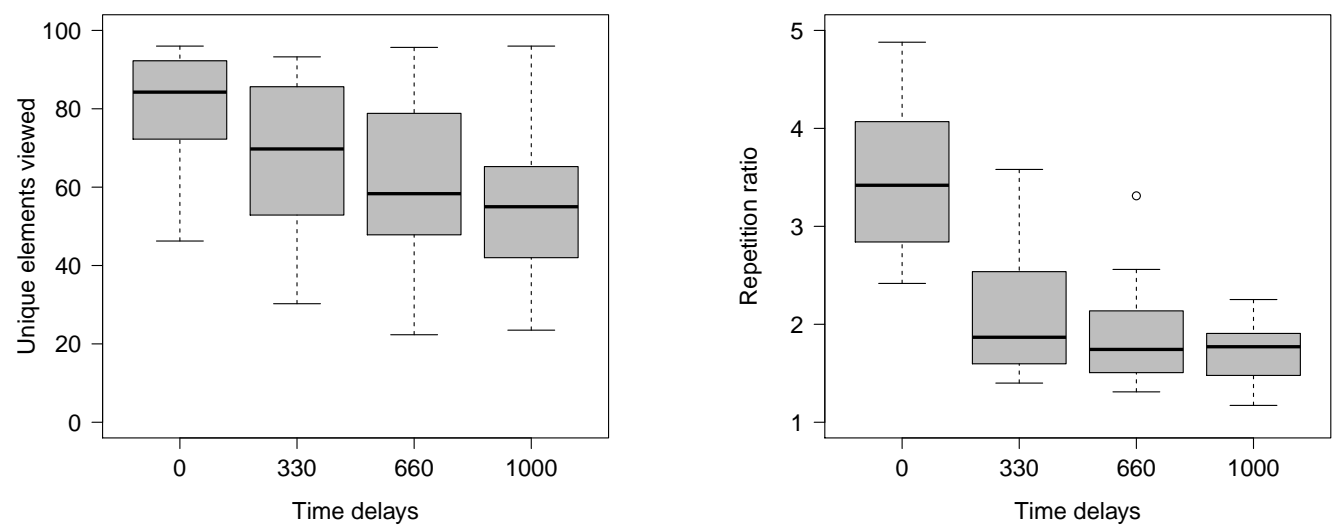

Both the number of unique elements (of 96 possible, left) and the repetition ratio, i.e. how often one information cell was opened on average (right), were significantly affected by increasing delay times.

(median: 70) information elements, this number was further reduced to 61 (median: 58 ) pieces of information in the $660 \mathrm{~ms}$ treatment. The difference between the $330 \mathrm{~ms}$ and $660 \mathrm{~ms}$ conditions was only marginally significant (osWT, $p=0.100$ ). Finally, in the $1000 \mathrm{~ms}$ treatment, on average 55 (median: 55) unique elements were revealed which is not significantly less than under the 660ms condition (osWT, $p=0.243$ ). Furthermore, the differences between $330 \mathrm{~ms}$ and $1000 \mathrm{~ms}$, $0 \mathrm{~ms}$ and $660 \mathrm{~m}$ as well as $0 \mathrm{~ms}$ and $1000 \mathrm{~ms}$ were all significant with $p=0.010, p<0.001$ and $p<0.001$, respectively (all osWT). These findings were supported by the regression analysis (see Table 2). In the discrete dummy specification, i.e. all delay treatments were compared to the baseline of $0 \mathrm{~ms}$, all delay dummy coefficients were significant. Using a cumulative dummy specification, i.e. measuring the marginal increase of $330 \mathrm{~ms}$ between two neighboring delay conditions, reveals that introducing delays $(0 \mathrm{~ms} \rightarrow 330 \mathrm{~ms}$ ) significantly reduced the number of revealed (unique) elements, but increasing delays from $330 \mathrm{~ms}$ to $660 \mathrm{~ms}$ or from $660 \mathrm{~ms}$ to $1000 \mathrm{~ms}$ did not.

RESUlT 4: Time delays only qualitatively changed the search index in the predicted direction.

EVIDENCE: In the absence of any time delay (0ms treatment) the average search index was -0.339 (median: -0.375) indicating a rather attribute-wise search pattern. Introducing time delays of $330 \mathrm{~ms}$ reduced the average search index to -0.374 
(median: -0.406), however the difference was not significant (osWT, $p=0.265$ ). Imposing a time delay of $660 \mathrm{~ms}$ further reduced the search index to an average of -0.445 (median: -0.505). Although the average again qualitatively shifts in the expected direction, the difference between $330 \mathrm{~ms}$ and $660 \mathrm{~ms}$ and between $0 \mathrm{~ms}$ and $660 \mathrm{~ms}$ was not significant at $p=0.266$ and only marginally significant at $p=0.069$ (both osWT), respectively. Further increasing the time delay to $1000 \mathrm{~ms}$ reduced the search index to an average of -0.492 (median: -0.478). Whereas the differences between $330 \mathrm{~ms}$ and $1000 \mathrm{~ms}$ as well as $660 \mathrm{~ms}$ and $1000 \mathrm{~ms}$ were not significant (both osWT with $p=0.152$ and $p=0.384$, respectively), participants used a significantly more attribute-wise search pattern in the $1000 \mathrm{~ms}$ treatment than without any delay (osWT, $p=0.038$ ). However, I could not find any statistically significant results in line with $\mathrm{H} 4$ in the regression analysis (see Table 2). Although all delay coefficients qualitatively led in the expected direction, none of them were significant.

RESULT 5: Different levels of time delay did not significantly affect the level of choice quality.

EVIDENCE: Remember that choice quality is a generic index computed as the utility level derived from the actual choice outcome divided by the utility derived from the most preferred outcome. In order to ensure comparability across product categories (see Figure 5), choice quality was 0-1 normalized for the further analysis. Under perfect information, a decision-maker is expected to choose the best alternative, i.e. the product from which she derives the highest level of utility (resulting in $\left.\theta=u_{n} / u_{\max }=1\right)$. Actually, in the absence of any time delay, participants on average achieved an outcome quality of 0.649 (median: 0.679 ). The average level of choice quality was reduced to an average of 0.651 (median: 0.603 ) in the $330 \mathrm{~ms}$ treatment. This difference is not significant (tsWT, $p=0.689$ ). Imposing time delays of $660 \mathrm{~ms}$ led to an average choice quality of 0.650 (median: 0.652 ). This level of choice quality is neither significantly different from the level at $0 \mathrm{~ms}$ nor at $330 \mathrm{~ms}$ (both tsWT, 0.569 and 0.631, respectively). Further increasing the time delay to $1000 \mathrm{~ms}$ resulted in an average choice quality of 0.741 (median: 0.731). Although seemingly larger, the average level of choice quality when imposing 1000ms was not significantly different from the quality levels under $0 \mathrm{~ms}, 330 \mathrm{~ms}$ or $660 \mathrm{~ms}$ treatment (all tsWT with $p=0.227, p=0.173$ and $p=0.227$, respectively). Conducting a regression analysis strongly supported the non-parametric results. Inducing search costs in the form of waiting times for information did not affect choice quality at all 
Table 2: Unique elements viewed and search index

\begin{tabular}{|c|c|c|c|c|c|c|c|c|}
\hline \multirow[b]{3}{*}{ Intercept } & \multicolumn{4}{|c|}{ Unique Elements Viewed } & \multicolumn{4}{|c|}{ Search Index } \\
\hline & \multicolumn{2}{|c|}{$\begin{array}{c}\text { discrete } \\
(1)\end{array}$} & \multicolumn{2}{|c|}{$\begin{array}{c}\text { cumulative } \\
(2)\end{array}$} & \multicolumn{2}{|c|}{$\begin{array}{c}\text { discrete } \\
(3)\end{array}$} & \multicolumn{2}{|c|}{$\begin{array}{c}\text { cumulative } \\
\text { (4) }\end{array}$} \\
\hline & $\begin{array}{c}103.905 \\
(10.646)\end{array}$ & $* * *$ & $\begin{array}{r}103.905 \\
(10.646)\end{array}$ & $* * *$ & $\begin{array}{r}-0.300 \\
(0.151)\end{array}$ & $*$ & $\begin{array}{r}-0.300 \\
(0.151)\end{array}$ & $*$ \\
\hline Delay $330 \mathrm{~ms}$ & $\begin{array}{r}-13.838 \\
(5.434)\end{array}$ & $*$ & $\begin{array}{r}-13.838 \\
(5.434)\end{array}$ & $*$ & $\begin{array}{r}-0.026 \\
(0.077)\end{array}$ & & $\begin{array}{r}-0.026 \\
(0.077)\end{array}$ & \\
\hline Delay $660 \mathrm{~ms}$ & $\begin{array}{c}-22.753 \\
(5.880)\end{array}$ & $* * *$ & $\begin{array}{r}-8.915 \\
(5.607)\end{array}$ & & $\begin{array}{r}-0.110 \\
(0.083)\end{array}$ & & $\begin{array}{r}-0.083 \\
(0.079)\end{array}$ & \\
\hline Delay $1000 \mathrm{~ms}$ & $\begin{array}{l}-26.166 \\
(5.580)\end{array}$ & $* * *$ & $\begin{array}{c}-3.413 \\
(5.768)\end{array}$ & & $\begin{array}{r}-0.129 \\
(0.079)\end{array}$ & & $\begin{array}{r}-0.019 \\
(0.081)\end{array}$ & \\
\hline Product type & $\begin{array}{r}2.397 \\
(1.681)\end{array}$ & & $\begin{array}{r}2.397 \\
(1.681)\end{array}$ & & $\begin{array}{r}0.067 \\
(0.026)\end{array}$ & $*$ & $\begin{array}{r}0.067 \\
(0.026)\end{array}$ & * \\
\hline Product costs & $\begin{array}{r}-1.147 \\
(3.512)\end{array}$ & & $\begin{array}{r}-1.147 \\
(3.512)\end{array}$ & & $\begin{array}{r}0.053 \\
(0.053)\end{array}$ & & $\begin{array}{r}0.053 \\
(0.053)\end{array}$ & \\
\hline Round & $\begin{array}{r}-4.630 \\
(0.725)\end{array}$ & $* * *$ & $\begin{array}{r}-4.630 \\
(0.725)\end{array}$ & $* * *$ & $\begin{array}{c}-0.005 \\
(0.011)\end{array}$ & & $\begin{array}{r}-0.005 \\
(0.011)\end{array}$ & \\
\hline Gross income & $\begin{array}{r}0.007 \\
(0.006)\end{array}$ & & $\begin{array}{r}0.007 \\
(0.006)\end{array}$ & & $\begin{array}{r}0.000 \\
(0.000)\end{array}$ & . & $\begin{array}{r}0.000 \\
(0.000)\end{array}$ & . \\
\hline Age & $\begin{array}{r}-0.599 \\
(0.381)\end{array}$ & & $\begin{array}{r}-0.599 \\
(0.381)\end{array}$ & & $\begin{array}{r}0.002 \\
(0.005)\end{array}$ & & $\begin{array}{r}0.002 \\
(0.005)\end{array}$ & \\
\hline Male & $\begin{array}{r}-2.582 \\
(3.977)\end{array}$ & & $\begin{array}{r}-2.582 \\
(3.977)\end{array}$ & & $\begin{array}{r}-0.035 \\
(0.056)\end{array}$ & & $\begin{array}{r}-0.035 \\
(0.056)\end{array}$ & \\
\hline Product costs $\times$ ross income & $\begin{array}{r}0.001 \\
(0.004)\end{array}$ & & $\begin{array}{r}0.001 \\
(0.004)\end{array}$ & & $\begin{array}{r}-0.000 \\
(0.000)\end{array}$ & & $\begin{array}{r}-0.000 \\
(0.000)\end{array}$ & \\
\hline Observations & 315 & & 315 & & 315 & & 315 & \\
\hline
\end{tabular}

Notes: Linear regressions with subject-specific random effects; heteroscedasticity-robust standard errors in parentheses; discretely specified delay dummies depict the dummy effect relative to no delay conditions (0ms); cumulatively specified delay dummies measure the difference between two neighboring delay conditions (e.g. 'delay 660ms' in a cumulative specification measures the delay effect between $660 \mathrm{~ms}$ and $330 \mathrm{~ms}$ ); including the incentivized measure of ambiguity aversion as well as other variables from a post-experimental questionnaire did not change the reported results; significance levels: ${ }^{* * *} p<0.001,{ }^{* *} p<0.01,{ }^{*} p<0.05$, and . $p<0.1$ 
(see also Table 3 and Figure 4).

Figure 4: Choice quality and choice efficiency
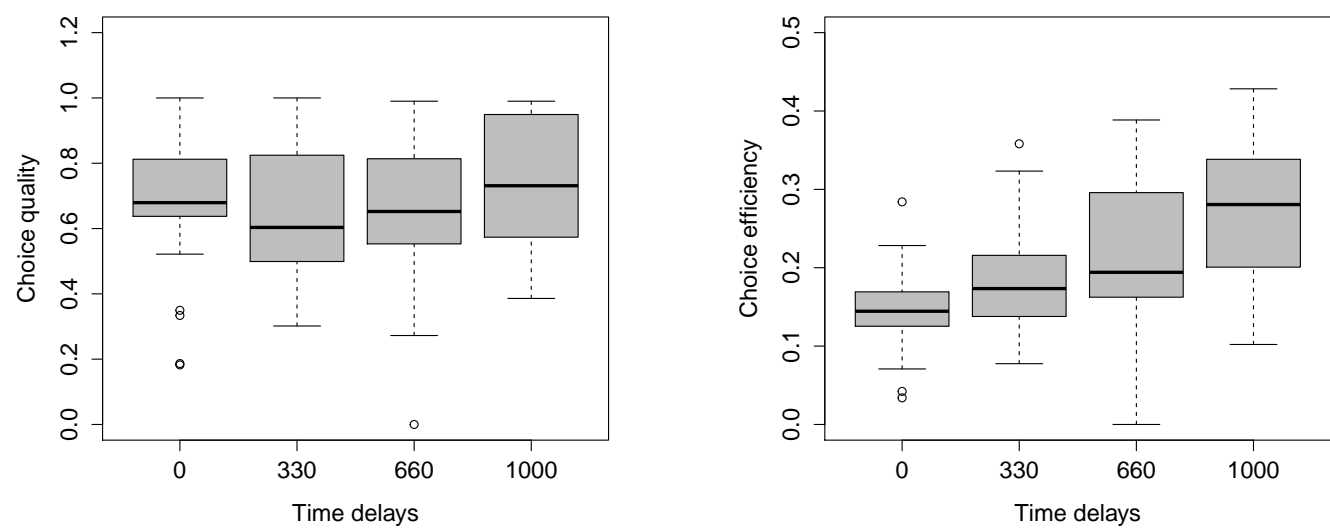

Whereas increasing delay times did not affect the levels of relative choice quality at all (left), they had a significant positive effect on choice efficiency, measured as choice quality per number of unique elements (right).

Remember that using a lexicographic search order means that information elements would be searched attribute-wise, starting with the most important attribute. In order to check whether the choice set consisting of real world products was exploitable by a lexicographic strategy at all, I analyzed the resulting hypothetical choice quality levels of lexicographically, processing only one and four of eight available attributes, respectively. Only looking-up the most important attribute (see Figure 5: LEX - 1 attribute only) resulted in an average hypothetical choice quality of 0.7434 (median: 0.818), which is significantly larger than the average choice quality under random choice (two-sided Wilcoxon Signed-Ranks Test, henceforth tsWT, $p<0.001)$ and even larger than the overall average empirically observed choice quality (tsWT, $p=0.065$ ). Increasing the number of processed attributes on average also improved choice quality (approaching unity if all attributes were revealed). ${ }^{34}$ Therefore, the actually used choice problems were potentially exploitable by applying a lexicographic search order. Furthermore, Figure 5 also depicts that participants in all product domains achieved significantly higher levels of choice

\footnotetext{
${ }^{34}$ However note that it is possible that looking-up fewer attributes results in a higher choice quality (see Figure 5: $\theta(\operatorname{top} 1)>\theta(t o p 4)$ ). This is because adding more information in a nondominated choice set could make some alternatives more attractive that would not otherwise be under perfect information.
} 
Table 3: Choice quality and choice efficiency

\begin{tabular}{|c|c|c|c|c|c|c|c|c|}
\hline \multirow[b]{3}{*}{ Intercept } & \multicolumn{4}{|c|}{ Choice Quality } & \multicolumn{4}{|c|}{ Choice efficiency } \\
\hline & \multicolumn{2}{|c|}{$\begin{array}{c}\text { discrete } \\
(1)\end{array}$} & \multicolumn{2}{|c|}{$\begin{array}{c}\text { cumulative } \\
(2)\end{array}$} & \multicolumn{2}{|c|}{$\begin{array}{c}\text { discrete } \\
(3)\end{array}$} & \multicolumn{2}{|c|}{$\begin{array}{c}\text { cumulative } \\
\text { (4) }\end{array}$} \\
\hline & 0.881 & $* * *$ & 0.881 & $* * *$ & 0.114 & & 0.114 & \\
\hline & $(0.137)$ & & $(0.137)$ & & $(0.069)$ & & $(0.069)$ & \\
\hline \multirow[t]{2}{*}{ Delay $330 \mathrm{~ms}$} & -0.038 & & -0.038 & & 0.046 & & 0.046 & \\
\hline & $(0.063)$ & & $(0.063)$ & & $(0.033)$ & & $(0.033)$ & \\
\hline \multirow[t]{2}{*}{ Delay $660 \mathrm{~ms}$} & -0.031 & & 0.007 & & 0.083 & $*$ & 0.037 & \\
\hline & $(0.072)$ & & $(0.069)$ & & $(0.035)$ & & $(0.034)$ & \\
\hline \multirow[t]{2}{*}{ Delay 1000ms } & 0.085 & & 0.116 & & 0.132 & $* * *$ & 0.048 & \\
\hline & $(0.065)$ & & $(0.071)$ & & $(0.034)$ & & $(0.035)$ & \\
\hline \multirow[t]{2}{*}{ Product type } & -0.043 & & -0.043 & & 0.013 & & 0.013 & \\
\hline & $(0.047)$ & & $(0.047)$ & & $(0.024)$ & & $(0.024)$ & \\
\hline \multirow[t]{2}{*}{ Product costs } & -0.044 & & -0.044 & & -0.040 & & -0.040 & \\
\hline & $(0.085)$ & & $(0.085)$ & & $(0.035)$ & & $(0.035)$ & \\
\hline \multirow[t]{2}{*}{ Round } & 0.026 & & 0.026 & & 0.034 & $* * *$ & 0.034 & $* * *$ \\
\hline & $(0.019)$ & & $(0.019)$ & & $(0.008)$ & & $(0.008)$ & \\
\hline \multirow[t]{2}{*}{ Gross income } & 0.000 & & 0.000 & & -0.000 & & -0.000 & \\
\hline & $(0.000)$ & & $(0.000)$ & & $(0.000)$ & & $(0.000)$ & \\
\hline \multirow[t]{2}{*}{ Age } & -0.011 & $*$ & -0.011 & $*$ & -0.002 & & -0.002 & \\
\hline & $(0.004)$ & & $(0.004)$ & & $(0.002)$ & & $(0.002)$ & \\
\hline \multirow[t]{2}{*}{ Male } & -0.038 & & -0.038 & & 0.007 & & 0.007 & \\
\hline & $(0.047)$ & & $(0.047)$ & & $(0.024)$ & & $(0.024)$ & \\
\hline \multirow[t]{2}{*}{ Product costs $\times$ gross income } & -0.000 & & -0.000 & & -0.000 & & -0.000 & \\
\hline & $(0.000)$ & & $(0.000)$ & & $(0.000)$ & & $(0.000)$ & \\
\hline Observations & 157 & & 157 & & 157 & & 157 & \\
\hline
\end{tabular}

Notes: Linear regressions with subject-specific random effect; heteroscedasticity-robust standard errors in parentheses; significance levels: ${ }^{* * *} p<0.001,{ }^{* *} p<0.01,{ }^{*} p<0.05$, and $. p<0.1$ 
quality than random choice would have resulted in (tsWT, $p<0.001) .^{35}$

Figure 5: Choice quality for hypothetical and observed levels of search intensity by product domain

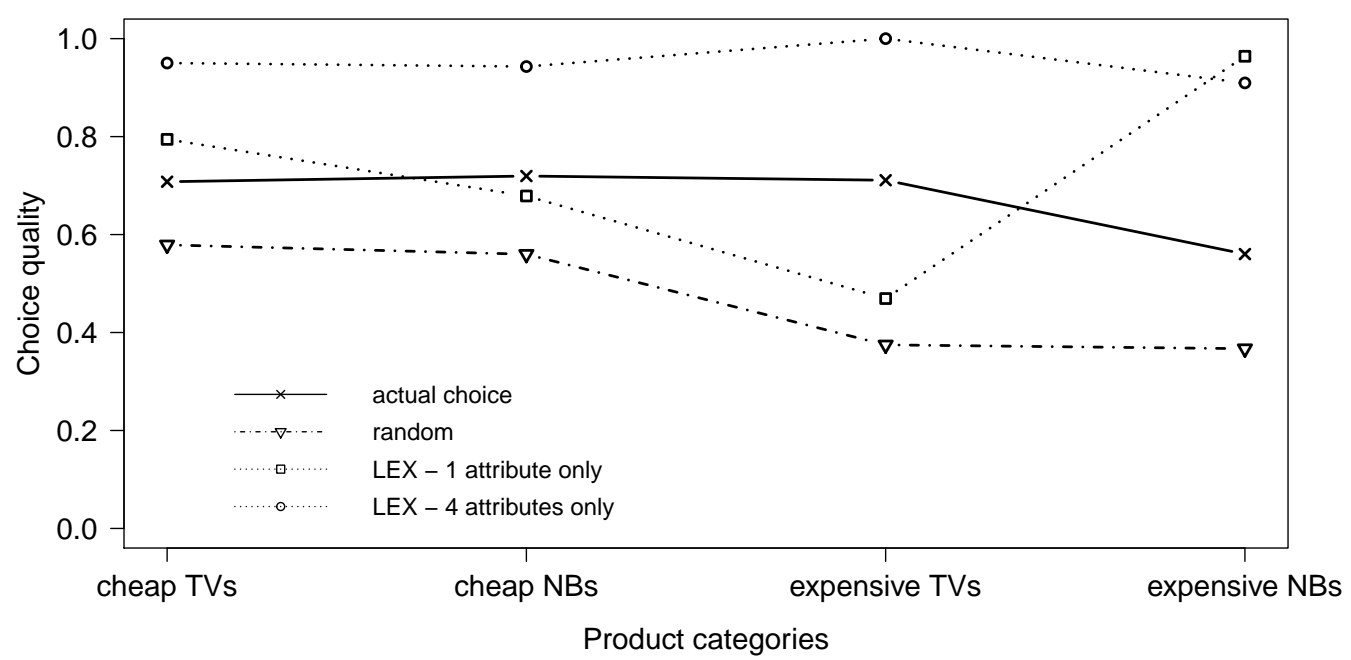

The solid line depicts the average quality of experimentally observed choices which is significantly higher than choosing randomly (dash-dotted line). The dotted lines display the levels of choice quality if only the most important attribute (squares) or the four most important attributes (circles) would have been used to choose a product. This procedure is similar to the one used in Fasolo et al. (2007).

RESULT 6: Increasing the levels of time delays led to a significant increase in choice efficiency.

EVIDENCE: I used a 0-1-normalized measure of choice efficiency to test H6. In the absence of time delays the average choice efficiency was 0.146 (median: 0.144). Imposing time delays of 330ms increased the average choice efficiency to 0.205 (median: 0.173). This difference is highly significant (osWT, $p=0.032$ ). Increasing the delay time to $660 \mathrm{~ms}$ resulted in an average choice efficiency of 0.233 (median: 0.194). The difference between the $0 \mathrm{~ms}$ and $660 \mathrm{~ms}$ condition was significant (osWT, $p=0.010$ ), but the difference between the $330 \mathrm{~ms}$ and $660 \mathrm{~ms}$ treatment was not (osWT, $p=0.202$ ). Finally, imposing $1000 \mathrm{~ms}$ time delays further increased the average choice quality to 0.275 (median: 0.281 ). The increase from $660 \mathrm{~ms}$ to $1000 \mathrm{~ms}$ was marginally significant (osWT, $p=0.055$ ) and both the differences between 0ms and $1000 \mathrm{~ms}$ and $330 \mathrm{~ms}$ and $1000 \mathrm{~ms}$ were highly significant (both osWT, with

\footnotetext{
${ }^{35}$ Note that the benchmark choice quality of choosing randomly was on average 0.484 (median: $0.468)$.
} 
$p<0.001$ and $p=0.002$, respectively). The regression analysis, in the discrete specification, also found significant time delay effects; however, in the cumulative specification, only the difference between $0 \mathrm{~ms}$ and $330 \mathrm{~ms}$ was marginally significant. This seemed to be a consequence of the definition of choice efficiency. Remember that $\eta=\theta / l$ and that there was identified a threshold level effect for the number of revealed elements regarding higher time delays (see Table 2, columns 3 and 4). Consequently, as neither choice quality, nor the number of information elements varied significantly between higher delay times, choice efficiency did not vary between higher levels of delay times either. Following the same reasoning, the significant round effect on choice efficiency was caused by the decrease in revealed information elements over time.

\section{Supplementary analysis}

\section{Search time per element}

Analyzing the duration an element was kept open before uncovering the next element revealed substantial differences between the delay time treatments. ${ }^{36}$ Participants on average spent 459ms (median: 435) to process one information element in the nodelay treatment. This processing time increased significantly (tsWT, $p<0.001$ ) to an average of $767 \mathrm{~ms}$ (median: 703 ) in the $330 \mathrm{~ms}$ delay treatment. Further increasing the delay time to $660 \mathrm{~ms}$ resulted in a significant rise in processing time per element (tsWT, $p=0.006$ ) to on average $879 \mathrm{~ms}$ (median: 819 ), which is also significantly larger than under the no-delay condition (tsWT, $p<0.001$ ). Imposing time delays of $1000 \mathrm{~ms}$ increased the processing time per element to on average $974 \mathrm{~ms}$ (median: 922), which is significantly larger than under the $0 \mathrm{~ms}$ or $330 \mathrm{~ms}$ delay conditions (both tsWT and $p<0.001$ ), but not significantly larger than in the 660ms delay treatment (tsWT, $p=0.153$ ). Furthermore, the length of the delay time and the time spent per element were strongly correlated (Spearman $r=0.694$ ).

\section{Repeated information acquisition}

In general, the participants made heavy use of the freedom to re-open any elements at any time. However, the repetition ratio (i.e. how often elements were re-opened

\footnotetext{
${ }^{36}$ Remember that only one element could be uncovered at a time. Moving the mouse cursor away from an element immediately re-covered the visible attribute values (irrespective of the delay treatment).
} 
on average) varied drastically between delay treatments (see Figure 3). Whereas one element was processed almost 4 times in the 0ms delay treatment (mean: 3.889, median: 3.419), the repetition ratio was almost halved when a marginal delay of 330ms was imposed (mean: 2.128, median: 1.868, tsWT: $p<0.001$ ). Increasing the delay time to $660 \mathrm{~ms}$ further decreased the repetition ratio (mean: 1.722, median: $1.668)$, resulting in a significant difference from the $0 \mathrm{~ms}$ condition $(p<0.001)$. However, the increase from $330 \mathrm{~ms}$ to $660 \mathrm{~ms}$ was not significant $(p=0.218)$. Imposing the maximum delay of $1000 \mathrm{~ms}$ further reduced the average repetition ratio to 1.697 (median: 1.771). However, this value was not significantly different from the $660 \mathrm{~ms}$ condition (tsWT, $p=0.361)^{37}$. Moreover, participants did not treat all information elements equally. The number of look-ups per attribute (including repetitions) was positively correlated with its respective attribute weight (Spearman, $r=0.172$ ), indicating that more important attributes were revealed more often.

\section{Pattern changes during the search process}

Figure 6: The search index for the first and second half of revealed elements

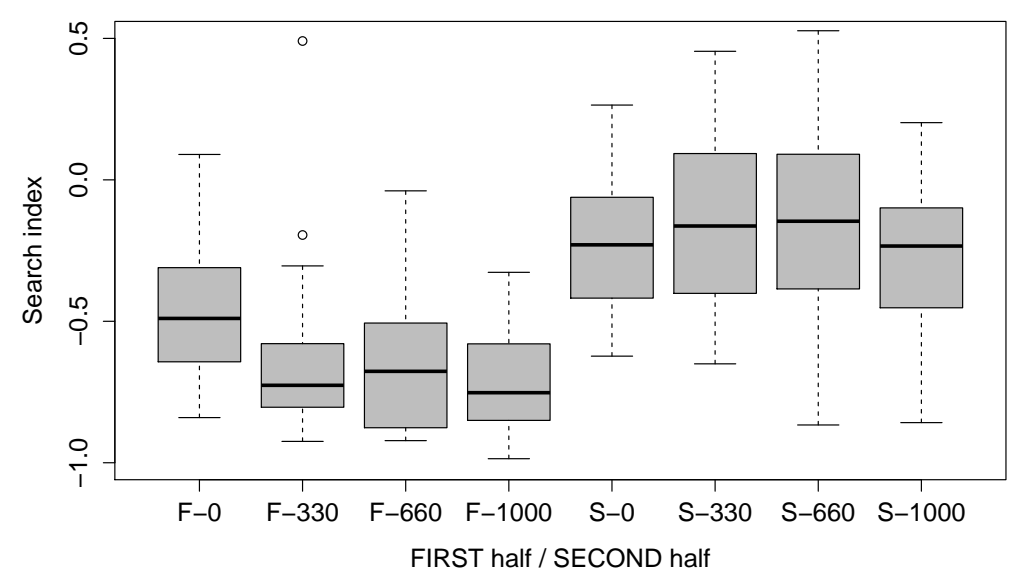

' $F$ ' denotes the first and ' $S$ ' the second half of all revealed elements (including repeated look-ups). In fact, the applied search patterns change significantly during the experimentally observed choice processes.

Splitting up the recorded process data to a first and second half of revealed elements (with repetitions, per choice problem) and analyzing them independently

\footnotetext{
${ }^{37}$ Whereas the difference between $0 \mathrm{~ms}$ and $1000 \mathrm{~ms}$ was highly significant (tsWT, $p<0.001$ ), the difference between $330 \mathrm{~ms}$ and $1000 \mathrm{~ms}$ was only marginally significant $(p=0.053)$.
} 
identified search pattern changes over time. Not surprisingly, almost all unique elements were viewed once during the first half (on average 80\%), leaving only very few pieces of information entirely unrevealed for the second half. Actually, only $17 \%$ of all unique elements were looked-up for the first time in the second half. ${ }^{38}$ Taking a closer look at the search index revealed an interesting change over time. Whereas the average search index for the first half was -0.626 , it increased substantially to -0.196 for the second half (see Figure 6). This difference was highly significant (osWT, $p<0.001$ ). Moreover, the variance of the number of elements looked-up per alternative (with repetitions) varied over time. ${ }^{39}$ Whereas it was on average 29 for the first half, it increased to 72 in the second half, meaning that search became more selective over time, focusing on a smaller subset of alternatives (osWT, $p<0.001)$. Consistent with the increasing variance in elements per alternative, I found a decreasing variance regarding the revealed elements per attribute over time (for TV sets and notebooks $p=0.022$ and $p=0.049$, respectively, both tsWT). Altogether this means that in the second half participants not only focused on fewer alternatives (reduced choice set) but also inspected this subset more closely (on more attributes) than in the first half. Finally, I found no changes in search pattern over time with respect to the time spent per element (all tsWT with $p>0.3$ ).

\section{Discussion}

\section{Opportunity costs of search}

Imposing search costs in the form of time delays in general affected information processing as predicted by the Effort-Certainty model. Hence, higher search costs, in line with previous results, significantly reduced the amount of information acquired, measured as both the number of unique elements and all elements including repeated look-ups (Payne et al. 1988, 1996, Kerstholt 1996, Dhar and Nowlis 1999, Rieskamp and Hoffrage 2008). However, as increasing time delays from $330 \mathrm{~ms}$ to $660 \mathrm{~ms}$ and from $660 \mathrm{~ms}$ to $1000 \mathrm{~ms}$ neither affected the number of unique elements looked-up nor the repetition ratio significantly (see Table 2, column 2), increasing time delays seemed to reduce the acquired amount of information in a non-linear way. It remains though for future research to clarify whether the magnitude of the effect of time

\footnotetext{
${ }^{38}$ On average $3 \%$ of all unique elements were disregarded altogether.

${ }^{39}$ Analyzing the variances of lookups per attribute or alternative is a standard approach in the strategy selection literature (see Payne et al. (1996), Bröder and Schiffer (2003)).
} 
delays above 330ms monotonically decreased with increasing levels of time delays or whether there existed a structural break, probably even resulting in choice deferral for unbearably high time delays, e.g. over 1000ms (see Dhar and Nowlis 1999).

In contrast to the search costs studies of Smith et al. (1982), Zakay and Wooler (1984), Payne et al. (1996), Kerstholt (1996) and Rieskamp and Hoffrage (2008), time delays overall seemed to affect the search index metric very little. However, I found a substantial change in the search index throughout the decision process that was not only highly significant but also very similar to previous findings in magnitude. Splitting up the recorded process data to a first and second half of revealed elements (with repetitions, per choice problem) and analyzing them independently identified search pattern changes over time. Similar to Verplanken (1993), who found a significant change in the search index from -0.52 to -0.28 , the current study identified a shift from -0.61 to -0.19 . Furthermore, consistent with the notion of forming consideration sets (e.g. Häubl and Trifts 2000, Mehta et al. 2003, Hauser 2010), the participants initially seemed to screen the full choice problem to get a general impression about the available choice options (more attribute-wise search in the first half). After building a consideration set of potentially suitable alternatives they seemed to switch to more intensively analyzing the remaining choice set (more alternative-wise search in the second half). This interpretation is also in line with the observed change of variance of information look-ups per alternative and per attribute. Whereas participants in the first half focused on a broader set of alternatives but fewer attributes, they changed their patterns to investigating fewer alternatives on more attributes in the second half, i.e. analyzing the products remaining in the reduced choice set in greater detail (see Payne et al. 1996, Bröder and Schiffer 2003).

The strong positive correlation between processing time per element and delay times (Spearman $r=0.694$ ) could have two potential causes. Schurman and Brutlag (2009), in a field study on the Internet search portals Google and bing, found that increasing the artificially imposed delay times caused a direct proportional increase in the users' response times to click on a search result ("time to click"). Similar to Schurman and Brutlag's results, I found that the delay times were strongly externally pacing participants, making them process information elements faster under low and slower under high time delays. This interpretation is also in line with management literature, which found that employees were significantly reacting to external pacers (Gersick 1994, Waller et al. 2002). However, alternatively, partici- 
pants could have deliberately used more time per element to memorize the revealed attribute value better (see Daneman and Carpenter 1980, Conway et al. 2005). This strategy would become more and more important when repeatedly looking-up information becomes increasingly more costly under higher delay times. Verplanken (1993) and Verplanken and Weenig (1993) for time pressure, and similarly Payne et al. (1996) for incentivized opportunity costs of time, found that increasing search costs reduced the processing time per information element. This suggests that there exist important differences between other search costs implementations and delay times. Most likely, this is because of the different incentives to deliberate on the selection of possible search strategies. Whereas in a situation with time delays (and also with monetary search costs) there are clear incentives to think twice about the next (costly) information look-up, in a situation of time pressure quite the opposite is true. Deliberating about the next move is costly, not the move itself. Consequently, it would only be rational to observe faster information processing for settings of indirectly imposed search costs such as time pressure, whereas just the contrary is true under direct cost conditions as used in the current study. Therefore, my results clearly emphasize that if information search costs are the dimension of interest, experimentally implementing them directly (instead of taking an indirect detour of artificially incentivizing the opportunity costs of time monetarily) is of crucial importance.

\section{Choice quality and efficiency}

In light of the above, it is not surprising that studies which imposed indirect search costs found that higher time pressure significantly reduced the levels of choice quality (Zakay and Wooler 1984, Benbasat and Dexter 1986, Payne et al. 1988, Hahn et al. 1992). However, implementing direct search costs in the form of delay times did not affect choice quality at all. Although high time delays made participants use fewer information elements, choice quality did not change across delay treatments. This result cannot be explained by random choice, as participants made choices of significantly better quality than random choice would have resulted in (see Figure 5). However, participants seem to have systematically exploited the structure of the choice environments.

Two properties could potentially be used to the decision-makers' advantage: a positive correlation of attributes and a sufficiently high dispersion in attribute 
weights (Bettman et al. 1998, Bröder 2000, Rieskamp and Otto 2006, Gigerenzer and Gaissmaier 2011). Attributes are deemed to be positively correlated if products that are good on one attribute also tend to be good on other attributes. A high positive correlation of attributes could then potentially be exploited by comparing all alternatives on just one (randomly selected) attribute, as the probability is relatively high (compared to zero of negative attribute correlations) that the product performing well on this single characteristic also performs well on others. In the context of this study, it is highly unlikely that attribute correlations were exploited as the attributes of the experimental choice sets were almost not correlated at all. Unlike Fasolo et al. (2007), where the inter-attribute correlations on average were 0.5, the correlation coefficients (Spearman) of the actually used choice environments of the present study were $-0.017,-0.067,-0.012$ and 0.024 for cheap TV sets, cheap notebooks, expensive TV sets and expensive notebooks, respectively.

As there were almost no attribute correlations to be exploited, the choice environment must have provided a sufficiently high dispersion of attribute weights and the participants must have used an — at least to some extent - lexicographic search order. Otherwise, they would not have been able to achieve relatively high levels of choice quality although using less information (in the high delay conditions). However, it is worth noting that attribute weights do not have to be extremely skewed. In the current experiment, the weight dispersion was rather moderate for all product domains but though led to a successful exploitation of this structure. ${ }^{40}$ In real choice environments, retailers or search sites usually describe products by a set of attributes that are commonly accepted to play a significant role (e.g. in the case of notebook computers: CPU-speed, RAM-capacity etc.). The extent to which choice efficiency could be increased by reducing the amount of looked-up information in such an environment depends on the dispersion of the relative weights of the commonly considered attributes.

\section{Product space variations}

The variation of product domains did not have a great influence on the tested set of metrics. Actually, the differences between the used treatment manipulations of cheap TV sets, cheap notebooks, expensive TV sets and expensive notebooks did not

\footnotetext{
${ }^{40}$ The attribute dispersion $\lambda$, as defined on page 10, on average was 0.120 and 0.211 for TV sets and notebooks, respectively.
} 
seem to be perceived 'different enough' to be considered as self-contained product domains. Even though I am not suggesting that the participants could not distinguish TV sets from notebooks, both products (and price levels) might have been perceived as belonging to one domain of 'medium to highly priced consumer electronics' and thereby diminish any differences between the actually used treatment manipulations. However, the obtained result does not necessarily mean that people disregard the importance of a choice situation as a relevant factor that affects adapting search intensity altogether. The questions of what constitutes the perception of a consumer product choice situation and what makes it perceived differently from others seem to outline an interesting avenue for future research.

The fact that participants used slightly more non-compensatory search patterns for notebooks than for TV sets might have been caused by a structural characteristic of the specific choice set itself. As I used unmodified characteristics of real world products, some alternatives had exactly the same values on certain attributes, meaning that looking-up these attribute values did not reveal information that could be used to discriminate between alternatives. Participants might have learned that this was the case for TV sets more often (e.g. all TV sets had a frequency of $100 \mathrm{~Hz}$ and all expensive TV sets additionally were capable of displaying full $H D$ resolution) and might have adjusted their search patterns accordingly. ${ }^{41}$

\section{Conclusion}

The primary goal of this paper is to investigate if and how slow connections, lags and waiting times for information in online environments affect information search and choice behavior and thus should not be neglected in the scholarly analysis of multi-attribute consumer choice. Therefore, this paper analyzed the effect of different levels of pure time delays on (i) information acquisition patterns, (ii) outcome quality and (iii) choice efficiency in a controlled laboratory setting. In line with the predictions of the Effort-Certainty model, increasing the delay times for providing information significantly reduced the amount of searched information elements;

\footnotetext{
${ }^{41}$ Anticipating that no discriminatory information is gained by looking-up e.g. the screen frequency of TV sets potentially reduces the number of alternative-wise transitions which resulted in a lower search index for TV sets. I deliberately neither modified the products' characteristics nor selected only such attributes in which all alternatives were different but those that were in general used by (online) retailers to describe the products in order to provide a more natural choice environment.
} 
however, interestingly, it did not change the quality of product choice at all. This suggests that the participants behaved highly adaptively and were able to exploit the structure of the choice environment. They seemed to have applied a lexicographic search order and started the search process by eliminating some alternatives on the basis of unsatisfactory attribute values on the most important characteristics and used the remaining alternatives to form a consideration set. Later on in the search process the remaining alternatives were investigated in more detail along the full range of attributes.

Moreover, higher time delays also made participants keep information elements open for a longer period before revealing the next element. Interestingly, this increase in deliberation time per element was not skewed towards the beginning or the end of a search process, making it unlikely that people developed a search plan upfront before starting to reveal information. In fact, it seems to be more probable that thinking about the next step (i.e. which information element to reveal next) dominated the observable search behavior. Although decision-makers reduced the number of information elements and thereby increased their choice efficiency as measured by choice quality per number of revealed elements, the total time to make a choice was not significantly different throughout all search cost treatments. One way of reducing the delay time induced increase of deliberation time per element, and thereby most likely increasing time-wise choice efficiency, could be to make consumers think about the overall search strategy first. Spending some time on deliberating what one wants or expects from a product of a specific domain and subsequently searching accordingly could save the time to reflect on 'which element to search next' for each element separately. It would therefore be interesting for future research to test ways of nudging decision-makers to reflect about their wants and a possibly appropriate search strategy before starting to search. Having clear expectations about the desired product and a rough plan about how to find it could reduce inefficient over-searching of the choice environment.

This study also has important implications for manufacturers and retailers of consumer durables. Which product is perceived to dominate others, and thus is finally chosen, not only depends on the consumer's preferences but also on the specific process of acquiring and integrating product information. My results indicate that time delays strongly affected how product information was processed and therefore could also have affected which product was chosen as a result of a specific search process. As delay times were manipulated between subjects, it was not possible to 
identify a direct 'product switching effect'; however, the significant changes in search behavior most likely affected the final product choice as well (see Branco et al. 2012). More specifically, consumers disregarded more than half of all attributes when experiencing high delay times. In such a situation, one expects that products are more likely to be chosen if they perform well on a few key characteristics. It then does not matter whether or not they perform well or badly on the other (non-key) attributes that are most likely not revealed anyway, under high time delay conditions. Under short time delays however, almost all attributes were processed before making the choice. Therefore, the profitability of the managerial decision, whether to concentrate manufacturing and sales resources on products that outperform their direct competitors on only a few attributes, or instead produce models that are more balanced across their attribute values, also depends on the speed of information provision of the used online retailing facilities. As technological advances are expected to further reduce delay times of online retailing platforms, products with balanced attribute values could be more successful as compared to those that are only superior regarding a few characteristics.

Although delay times did not affect participants' choices regarding the retailers' revenue, providing slow online platforms could have severe consequences of a different kind. This study only investigated the effect of waiting times under monopolistic conditions, i.e. subjects did not have the opportunity to switch to an alternative retailer. As already suggested by Schurman and Brutlag (2009), high delay times could have severe negative implications on the profitability of a search platform provider, when there are alternative providers available. However, investigating the effect of time delays on the competitiveness of online retailers is beyond the scope of this study and remains an interesting opportunity for future research.

\section{Acknowledgments}

I would like to thank Florian Artinger, Morten Hviid, Konstantinos Katsikopoulos, Abhijit Ramalingam, Stephan Schosser, Michael Schulte-Mecklenbeck, Robert Sugden, and Daniel Zizzo for helpful comments and suggestions. I also thank the participants of the Workshop on Testing Theories of Choice Behavior (Berlin, Germany, 2012), the annual meeting of the German Association of Experimental Economics (Karlsruhe, Germany, 2012), and the ECO research seminar of the School of 
Economics, University of East Anglia (Norwich, UK, 2012) for their comments and suggestions. Furthermore, I acknowledge the financial support in form of a scholarship grant (Förderstipendium) of the Vienna University of Economics and Business. The usual disclaimer applies. The data is available from the author.

\section{References}

Abendroth, Lisa J, Kristin Diehl. 2006. Now or Never: Effects of Limited Purchase Opportunities on Patterns of Regret over Time. Journal of Consumer Research 33(3) 342-351.

Benbasat, Izak, Albert S Dexter. 1986. An Investigation of the Effectiveness of Color and Graphical Information Presentation under Varying Time Constraints. MIS Quarterly 10(1) 59-83.

Bettman, James R, Mary Frances Luce, John W Payne. 1998. Constructive Consumer Choice Processes. Journal of Consumer Research 25(3) 187-217.

Birnbaum, Michael, Ulrich Schmidt. 2008. An experimental investigation of violations of transitivity in choice under uncertainty. Journal of Risk and Uncertainty 37(1) $77-91$.

Böckenholt, Ulf, Linda S. Hynan. 1994. Caveats on a process-tracing measure and a remedy. Journal of Behavioral Decision Making 7(2) 103-117.

Branco, F., M. Sun, J. M. Villas-Boas. 2012. Optimal Search for Product Information. Management Science 58(11) 2037-2056.

Bröder, Arndt. 2000. Assessing the empirical validity of the "Take-the-best" heuristic as a model of human probabilistic inference. Journal of Experimental Psychology: Learning, Memory, and Cognition 26(5) 1332-1346.

Bröder, Arndt. 2003. Decision Making With the "Adaptive Toolbox": Influence of Environmental Structure, Intelligence, and Working Memory Load. Journal of Experimental Psychology - Learning, Memory, and Cognition 29(4) 611-625.

Bröder, Arndt, Stefanie Schiffer. 2003. Bayesian strategy assessment in multi-attribute decision making. Journal of Behavioral Decision Making 16(3) 193-213.

Caplin, Andrew, Mark Dean, Daniel Martin. 2011. Search and Satisficing. American Economic Review 101(7) 2899-2922.

Chu, P C, Eric E Spires. 2000. The joint effects of effort and quality on decision strategy choice with computerized decision aids. Decision Science 31(2) 259-292. 
Conway, Andrew R A, Michael J Kane, Michael F Bunting, Zach D Hambrick, Oliver Wilhelm, Randall W Engle. 2005. Working memory span tasks: A methodological review and users guide. Psychonomic Bulletin \& Review 12(5) 769-786.

Coupey, Eloise, Sunder Narayanan. 1996. Effects of Knowledge Types on Choice Quality and Perceptions of Choice Performance. Psychology and Marketing 13(October) $715-738$.

Cox, James C, Vjollca Sadiraj, Ulrich Schmidt. 2011. Paradoxes and Mechanisms for Choice under Risk. Working Paper 1-44.

Czerlinski, Jean, Gerd Gigerenzer, Daniel G Goldstein. 1999. How good are Simple Heuristics? Gerd Gigerenzer, Peter M Todd, eds., Simple heuristics that make us smart, 1st ed., chap. 5. Oxford Univ. Press, Oxford et al., 97-118.

Daneman, Meredyth, Patricia A Carpenter. 1980. Individual Differences in Working Memory and Reading. Journal of Verbal Learning and Verbal Behavior 19 450-466.

Dhar, Ravi, Stephen M Nowlis. 1999. The Effect of Time Pressure on Consumer Choice Deferral. Journal of Consumer Research 25(4) 369-384.

Diehl, Kristin. 2005. When Two Rights Make a Wrong: Searching Too Much in Ordered Environments. Journal of Marketing Research 42(3) 313-322.

Fasolo, Barbara, Gary H McClelland, Peter M Todd. 2007. Escaping the Tyranny of Choice: When fewer attributes make choice easier. Marketing Theory 7(1) 13-26.

Gersick, Connie JG. 1994. Pacing Strategic Change: The Case of a New Venture. The Academy of Management Journal 37(1) 9-45.

Gigerenzer, Gerd, Henry Brighton. 2009. Homo Heuristicus: Why Biased Minds Make Better Inferences. Topics in Cognitive Science 1(1) 107-143.

Gigerenzer, Gerd, Wolfgang Gaissmaier. 2011. Heuristic Decision Making. Annual Review of Psychology 62 451-82.

Hahn, Minhi, Robert Lawson, Young Gyu Lee. 1992. The Effects of Time Pressure and Information Load on Decision Quality. Psychology and Marketing 9(5) 365-378.

Häubl, Gerald, Valerie Trifts. 2000. Consumer Decision Making in Online Shopping Environments: The Effects of Interactive Decision Aids. Marketing Science 19(1) 4-21.

Hauser, John R. 2010. Consideration-Set Heuristics. Working Paper .

Inman, J Jeffrey, Marcel Zeelenberg. 2002. Regret in Repeat Purchase versus Switching Decisions: The Attenuating Role of Decision Justifiability. Journal of Consumer Research 29(1) 116-128.

Johnson, Eric J, John W Payne. 1985. Effort and Accuracy in Choice. Management Science 31(4) 395-414. 
Johnson, Eric J, John W Payne, D A Schkade, James R Bettman. 1986. Monitoring Information Processing and Decisions: The Mouselab System. Tech. rep., Duke University Durham NC Center for Decision Studies.

Katsikopoulos, Konstantinos V, Lael J Schooler, Ralph Hertwig. 2010. The Robust Beauty of Ordinary Information. Psychological Review 117(4) 1259-1266.

Kerstholt, José H. 1996. The effect of information costs on strategy selection in dynamic tasks. Acta Psychologica 94(3) 273-290.

Klein, Noreen M, Manjit S Yadav. 1989. Context Effects on Effort and Accuracy in Choice: An Enquiry into Adaptive Decision Making. Journal of Consumer Research 15(4) 411-421.

Kruglanski, Arie W, Gerd Gigerenzer. 2011. Theoretical Notes Intuitive and Deliberate Judgments Are Based on Common Principles. Psychological Review 118(1) 97-109.

Kuo, Feng-Yang, Tsai-Hsin Chu, Meng-Hsiang Hsu, Hong-Ssu Hsieh. 2004. An investigation of effort-accuracy trade-off and the impact of self-efficacy on Web searching behaviors. Decision Support Systems 37(3) 331-342.

Lee, Byung-Kwan, Wei-Na Lee. 2004. The effect of Information Overload on Consumer Choice Quality in an On-Line Environment. Psychology and Marketing 21(3) 159183.

Loomes, Graham, Robert Sugden. 1982. Regret Theory: An Alternative Theory of Rational Choice Under Uncertainty. The Economic Journal 92(368) 805-824.

Loomes, Graham, Robert Sugden. 1986. Disappointment and Dynamic Consistency in Choice under Uncertainty. The Review of Economic Studies 53(2) 271-282.

Mehta, Nitin, Surendra Rajiv, Kannan Srinivasan. 2003. Price Uncertainty and Consumer Search: A Structural Model of Consideration Set Formation. Marketing Science 22(1) $58-84$.

Payne, John W. 1976. Task Complexity and Contingent Processing in Decision Making: An Information Search and Protocol Analysis. Organizational Behavior and Human Performance 16(2) 366-387.

Payne, John W, James R Bettman, Eric J Johnson. 1988. Adaptive Strategy Selection in Decision-Making. Journal of Experimental Psychology-Learning Memory and Cognition 14(3) 534-552.

Payne, John W, James R Bettman, Eric J Johnson. 1993. The Adaptive Decision Maker. Cambridge University Press, Cambridge.

Payne, John W, James R Bettman, Mary Frances Luce. 1996. When Time Is Money: 
Decision Behavior under Opportunity-Cost Time Pressure. Organizational Behavior and Human Decision Processes 66(2) 131-152.

Reisen, Nils, Ulrich Hoffrage, Fred W Mast. 2008. Identifying decision strategies in a consumer choice situation. Judgement and Decision Making 3(8) 641-658.

Riedl, René, Eduard Brandstätter. 2007. Die Strategie der Entscheidung - Alte Probleme und neue Lösungen. NeuroPsychoEconomics 2(1) 59-75.

Rieskamp, Jörg, Ulrich Hoffrage. 2008. Inferences under time pressure: How opportunity costs affect strategy selection. Acta Psychologica 127 258-276.

Rieskamp, Jörg, Philipp E Otto. 2006. SSL: A theory of how people learn to select strategies. Journal of Experimental Psychology - General 135(2) 207-236.

Schurman, Eric, Jake Brutlag. 2009. Performance Related Changes and their User Impact. URL http://velocityconf.com/velocity2009/public/schedule/detail/8523.

Shao, Wei, Ashley Lye, Sharyn Rundle-Thiele. 2008. Decisions, decisions, decisions: Multiple pathways to choice. International Journal of Market Research 50(6) 797-816.

Simon, Herbert Alexander. 1956. Rational Choice and the Structure of the Environment. Psychological Review 63(2) 129-138.

Smith, James F, Terence R Mitchell, Lee Roy Beach. 1982. A Cost-Benefit Mechanism for Selecting Problem-solbing Strategies: Some Extensions and Empirical Tests. Organizational Behavior and Human Performance 29 370-396.

Stigler, George J. 1961. The Economics of Information. Journal of Political Economy 69(3) 213-225.

Tsiros, Michael, Vikas Mittal. 2000. Regret: A Model of Its Antecedents and Consequences in Consumer Decision Making. Journal of Consumer Research 26(4) 401-417.

Tversky, Amos. 1972. Choice by elimination. Journal of Mathematical Psychology 9(4) 341-367.

Urbany, Joel E. 1986. Experimental Examination of of the Economics Assumption of Prior Knowledge. Journal of Consumer Research 13(2) 257-271.

Verplanken, Bas. 1993. Need for Cognition and External Information Search: Responses to Time Pressure during Decision-Making. Journal of Research in Personality $\mathbf{2 7}$ $238-252$.

Verplanken, Bas, Mieneke W H Weenig. 1993. Graphical energy labels and consumers' decisions about home appliances: A process tracing approach. Journal of Economic Psychology 14(4) 739-752.

Waller, Mary J, Mary E Zellmer-bruhn, Robert C Giambatista. 2002. Watching the Clock: 
Group Pacing Behavior under Dynamic Deadlines. The Academy of Management Journal 45(5) 1046-1055.

Willemsen, Martijn C, Eric J Johnson. 2004. Mouselab. Tech. rep., Columbia University, New York. URL http://www . mouselabweb.org/.

Willemsen, Martijn C, Eric J Johnson. 2011. Visiting the decision factory: observing cognition with MouselabWEB and other information acquisition methods. Michael Schulte-Mecklenbeck, Anton Kühberger, R Ranyard, eds., A handbook of process tracing methods for decision research: a critical review and user's guide. Psychology Press, New York, 19-42.

Zakay, Dan, Stuart Wooler. 1984. Time pressure, training and decision effectiveness. Ergonomics 27(3) 273-284.

Zillow.com. 2010. Despite Mortgage Meltdown, Today's Borrowers Continue to Spend Twice as Much Time Researching a Car Purchase as Researching Their Home Loan. URL http://zillow. mediaroom. com/index . php?s=159\&item=201. 